Supporting Information:

\title{
A new Ir bis-carbonyl precursor for water oxidation catalysis
}

Daria L. Huang, Rodrigo Beltrán-Suito, Julianne M. Thomsen, Sara M. Hashmi†, Kelly L. Materna, Stafford W. Sheehan $\ddagger$, Brandon Q. Mercado, Gary W. Brudvig*, Robert H. Crabtree*

$†$ Department of Chemical and Environmental Engineering, Yale University, 9 Hillhouse Avenue, New Haven, Connecticut, 06520, United States

†Catalytic Innovations LLC, P.O. Box 356, Adamsville, Rhode Island, 02801, United States

*Department of Chemistry, Yale University, 225 Prospect Street, New Haven, Connecticut 06520, United States 


\section{Contents}

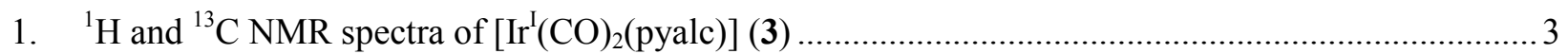

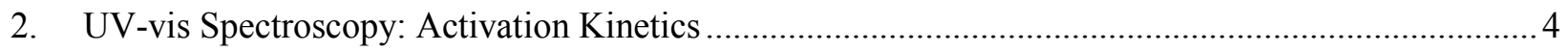

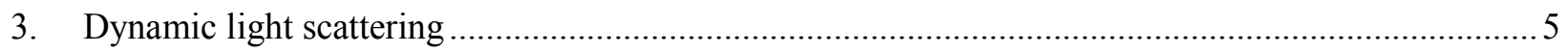

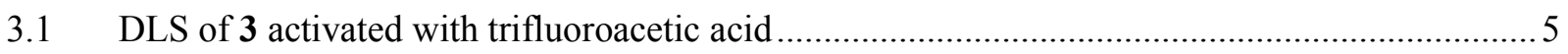

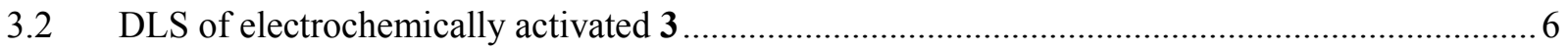

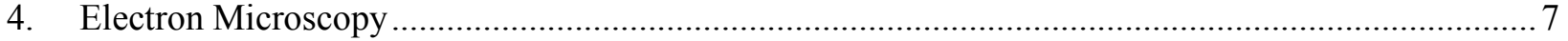

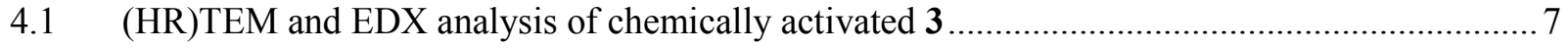

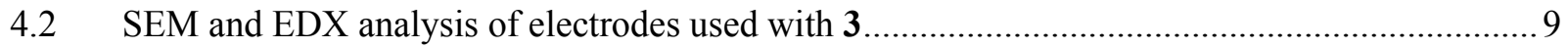

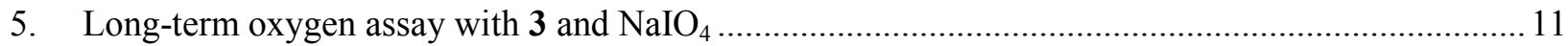

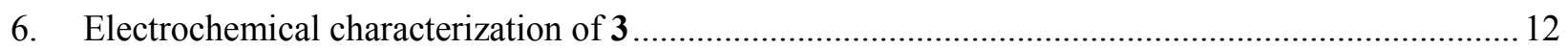

7. ${ }^{1}$ H NMR spectra of 2, 2', and 2'e

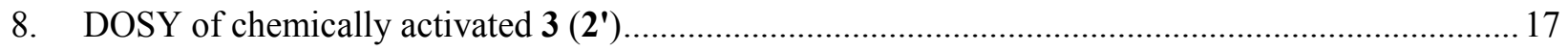

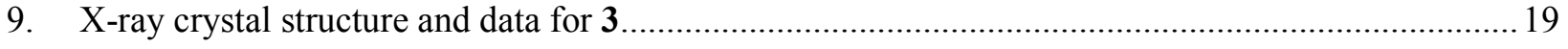

10. X-ray crystal structure and data for 3 co-crystallized with 1,4-butanediol ..................................27

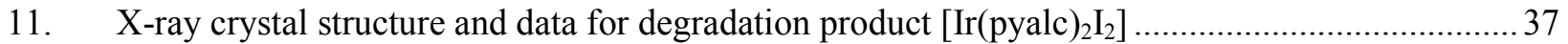


1. ${ }^{1} \mathrm{H}$ and ${ }^{13} \mathrm{C}$ NMR spectra of $\left[\operatorname{Ir}^{\mathrm{I}}(\mathrm{CO})_{2}(\right.$ pyalc $\left.)\right](3)$
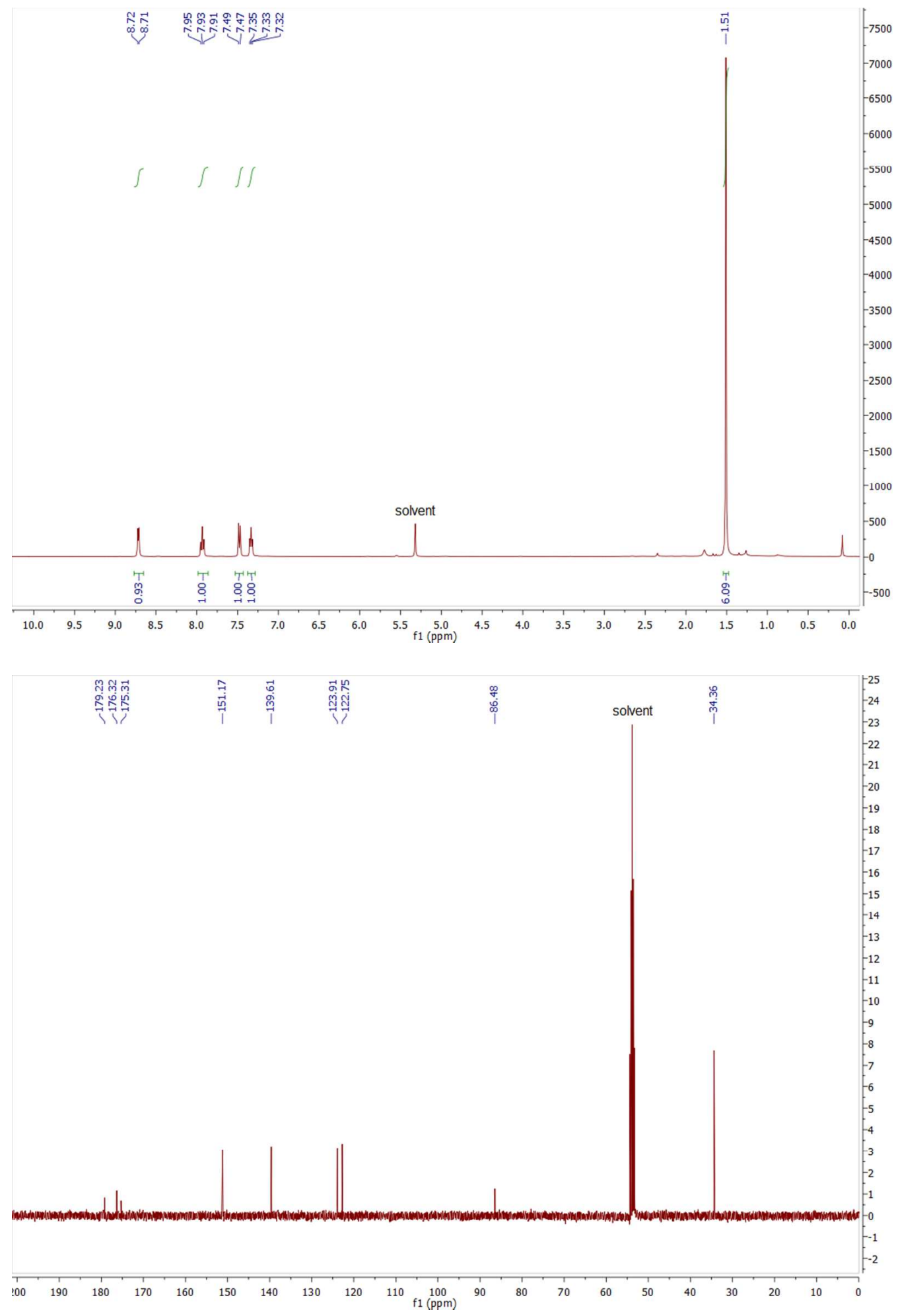

Figure S1. ${ }^{1} \mathrm{H}$ and ${ }^{13} \mathrm{C}$ NMR spectra of $\left[\operatorname{Ir}^{\mathrm{I}}(\mathrm{CO})_{2}\right.$ (pyalc)], both taken in $\mathrm{CD}_{2} \mathrm{Cl}_{2}$. 


\section{UV-vis Spectroscopy: Activation Kinetics}

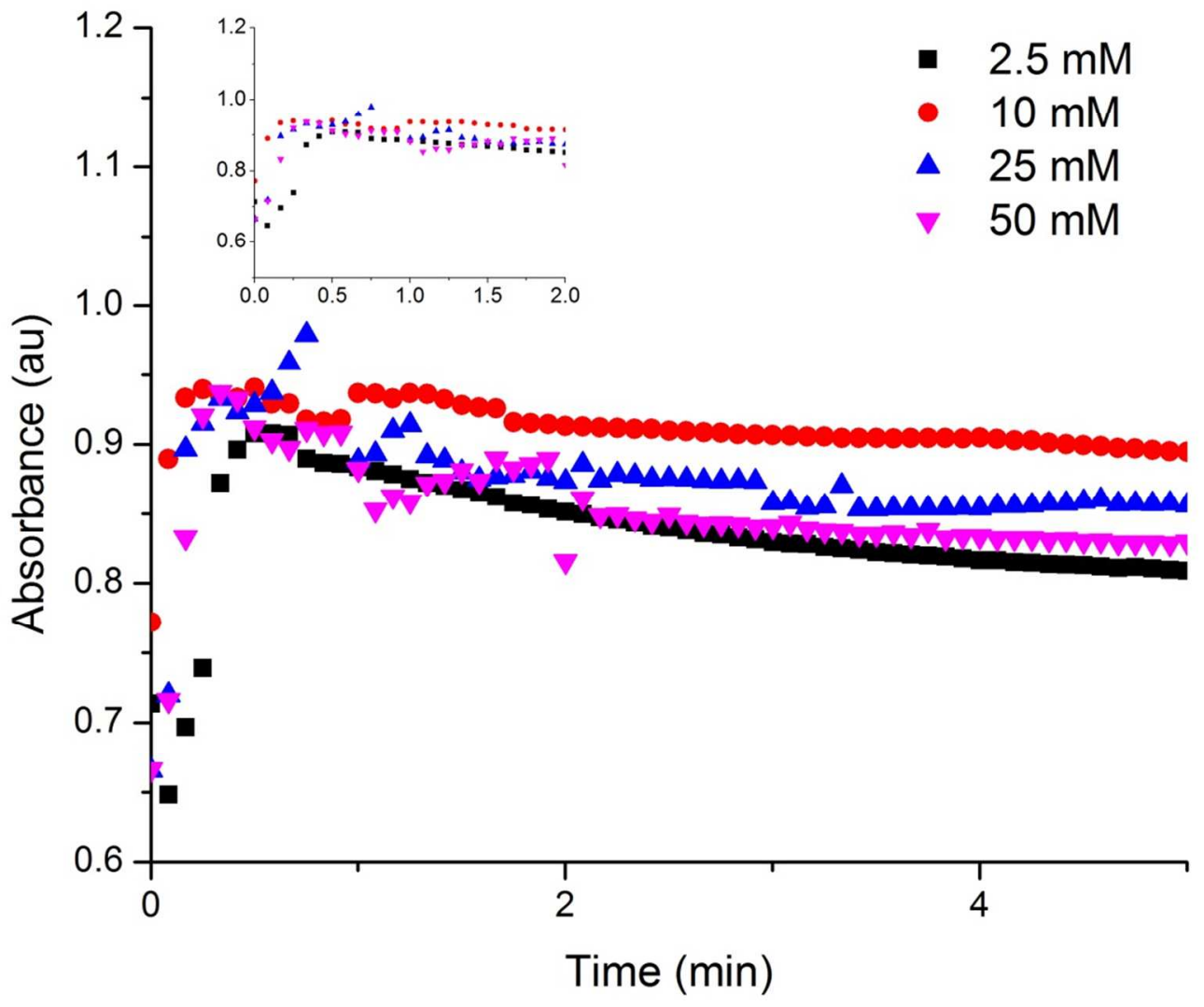

Figure S2. UV-vis kinetics of $0.5 \mathrm{mM}$ of 3 with various equivalents of $\mathrm{NaIO}_{4}$ (see legend) and $25 \mathrm{mM}$ acetic acid in $\mathrm{H}_{2} \mathrm{O}$, monitored at $595 \mathrm{~nm}$. The data show that 3 activates fully after $\sim 20$ seconds upon addition of oxidant (see inset), regardless of oxidant concentration. Decrease in absorbance after initial activation is due to interference from and eventual dissipation of bubbles (from water oxidation).

The data suggest that $\mathbf{3}$ activates with apparent zero-order kinetics. This differs from the Cp*Ir analogues, ${ }^{1}$ which have been shown to activate much more slowly and with first-order kinetics. This difference is likely due to the placeholder ligands (two CO vs. $\mathrm{Cp}^{*}$ ) and the ease at which they are lost during the activation process.

\footnotetext{
${ }^{1}$ Hintermair, U.; Sheehan, S. W.; Parent, A. R.; Ess, D. H.; Richens, D. T.; Vaccaro, P. H.; Brudvig, G. W.; Crabtree, R. H. J. Am. Chem. Soc. 2013, 135 (29), 10837-10851.
} 


\section{Dynamic light scattering}

3.1 DLS of 3 activated with trifluoroacetic acid

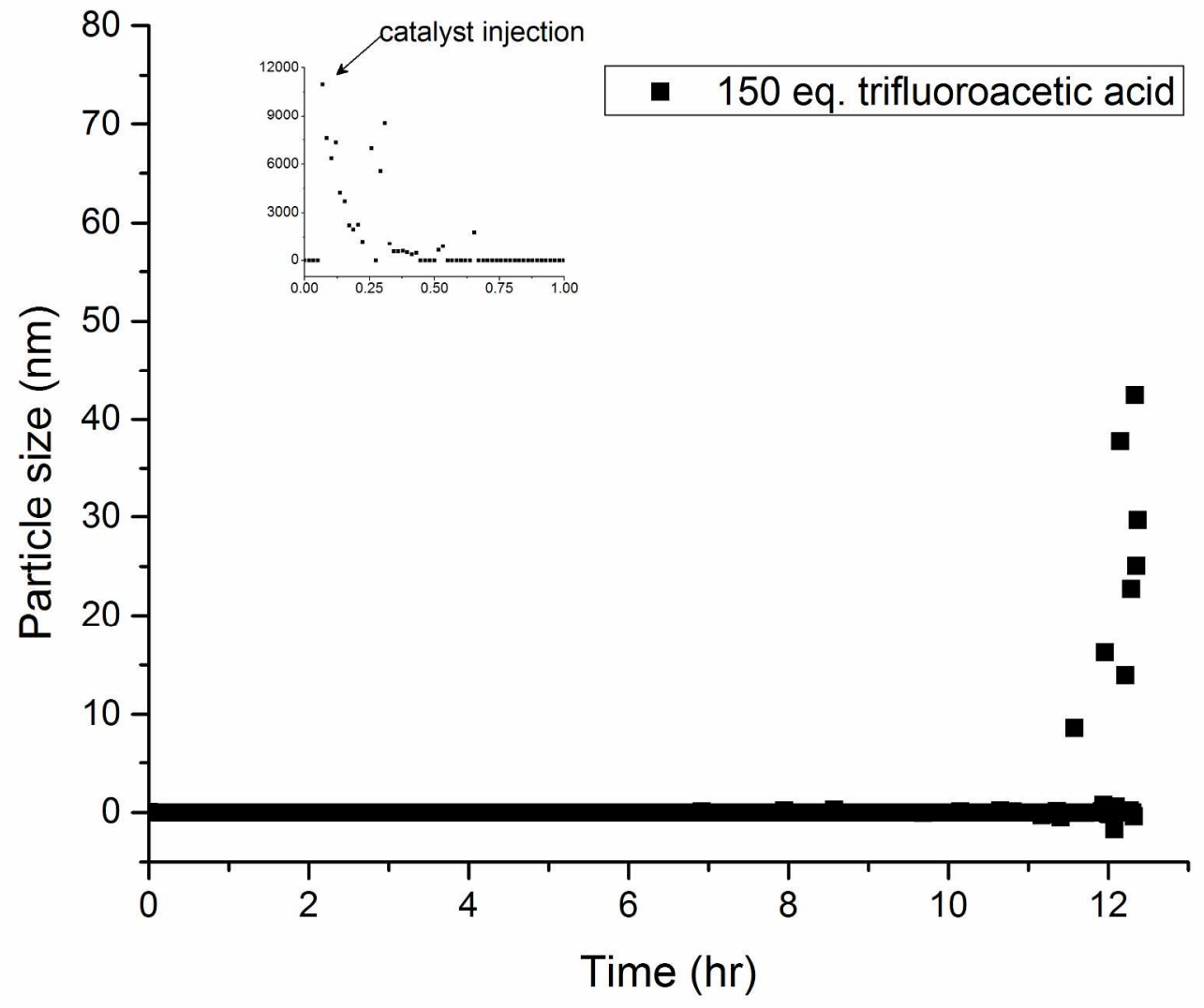

Figure S3. Mean particle size of a $4 \mathrm{~mL}$ solution of $0.25 \mathrm{mM} 3$ with 150 equivalents of trifluoroacetic acid and $250 \mathrm{mM} \mathrm{NaIO}_{4}$. Samples were passed through a $0.2 \mu \mathrm{m}$ PTFE filter prior to measurements. Small nanoparticles $(\sim 50 \mathrm{~nm})$ begin to form after 11 hours. Inset shows the large particle signals due to bubbles formed by initial water oxidation once catalyst is injected. 
3.2 DLS of electrochemically activated 3

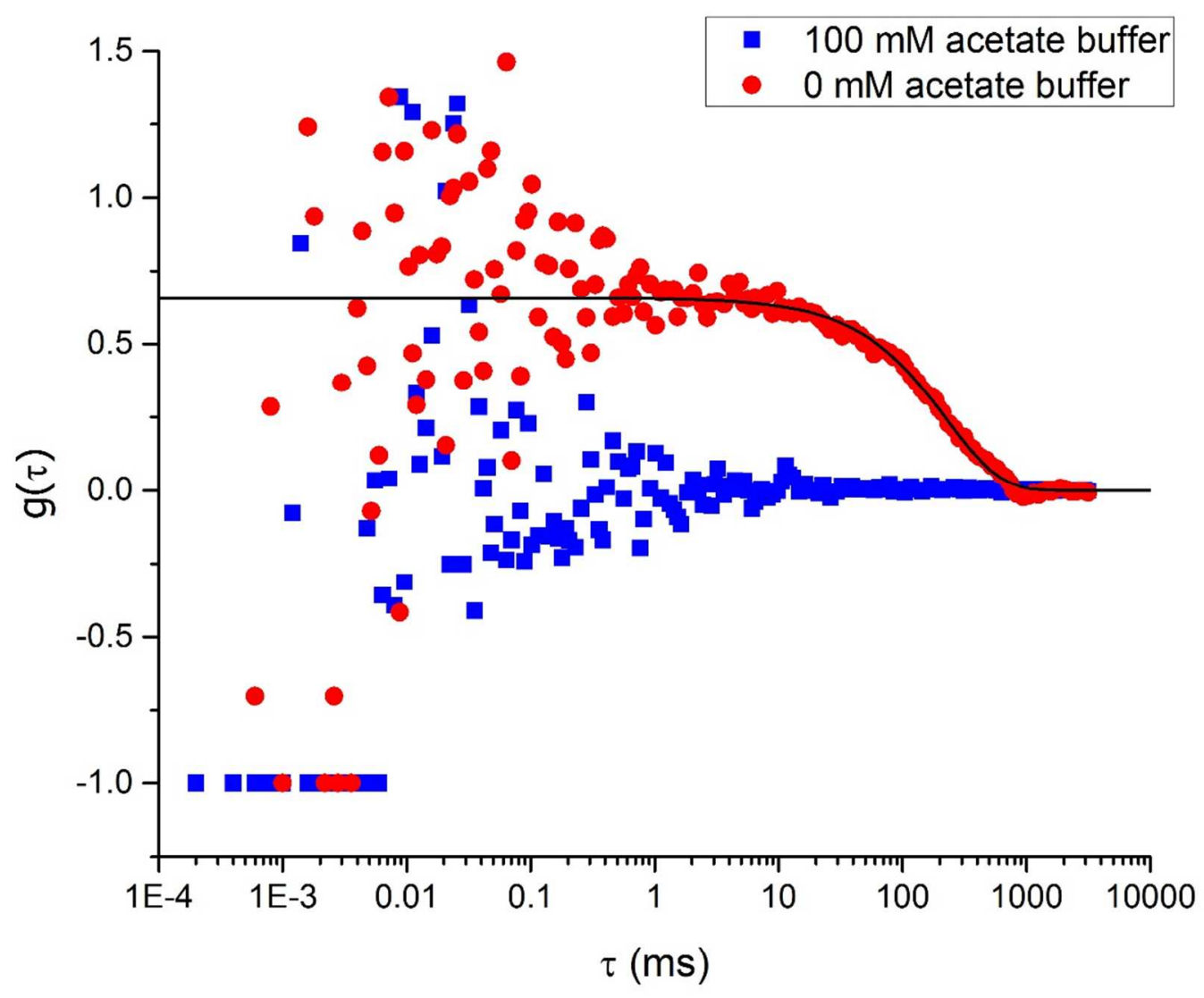

Figure S4. Dynamic light scattering correlation functions. Blue: $1 \mathrm{mM} \mathbf{2}^{\prime} \mathbf{e}$ in $0.25 \mathrm{M} \mathrm{Na}_{2} \mathrm{SO}_{4}$ and $100 \mathrm{mM}$ acetate buffer. Red: $1 \mathrm{mM} \mathbf{2}^{\prime} \mathbf{e}$ in $0.25 \mathrm{M} \mathrm{Na}_{2} \mathrm{SO}_{4}$ only. Samples were taken directly after water oxidation experiments and passed through a $0.2 \mu \mathrm{m}$ PTFE filter prior to measurements.

We have previously described particle size correlation calculations in a previous paper. ${ }^{2}$ The red trace displays a single diffusive relaxation time indicative of nanoparticles. This is fit to an exponential decay, $g(\tau)=\mathrm{a} \exp (-\tau / \mathrm{t})$, where $\mathrm{a}=0.66$ and $\mathrm{t}=230 \mathrm{~ms}$. The fit parameters suggest the presence of large aggregates, greater than 10 microns, which can appear after the formation of primary particles. The blue data, however, do not exhibit an exponential decay, suggesting that the acetate prevents the formation of nanoparticles during electrochemical water oxidation.

\footnotetext{
${ }^{2}$ Hintermair, U.; Hashmi, S. M.; Elimelech, M.; Crabtree, R. H. J. Am. Chem. Soc. 2012, 134 (23), 9785-9795.
} 


\section{Electron Microscopy}

\section{1 (HR)TEM and EDX analysis of chemically activated $\mathbf{3}$}

Transmission electron microscope (TEM) images were taken using an FEI Tecnai Osiris $200 \mathrm{kV}$ TEM. Samples were prepared by flowing aqueous suspensions of the iridium-containing solutions through copper TEM grids, supported by ultrathin carbon on a lacey carbon support or silicon monoxide (Ted Pella). The samples shown below use $\operatorname{Ir}(\mathrm{CO})_{2}$ (pyalc) at a concentration of $2 \mathrm{mg} / \mathrm{mL}$ in water, oxidized with $\mathrm{NaIO}_{4}$, and were applied to the TEM grids immediately after formation of the blue-colored active species.

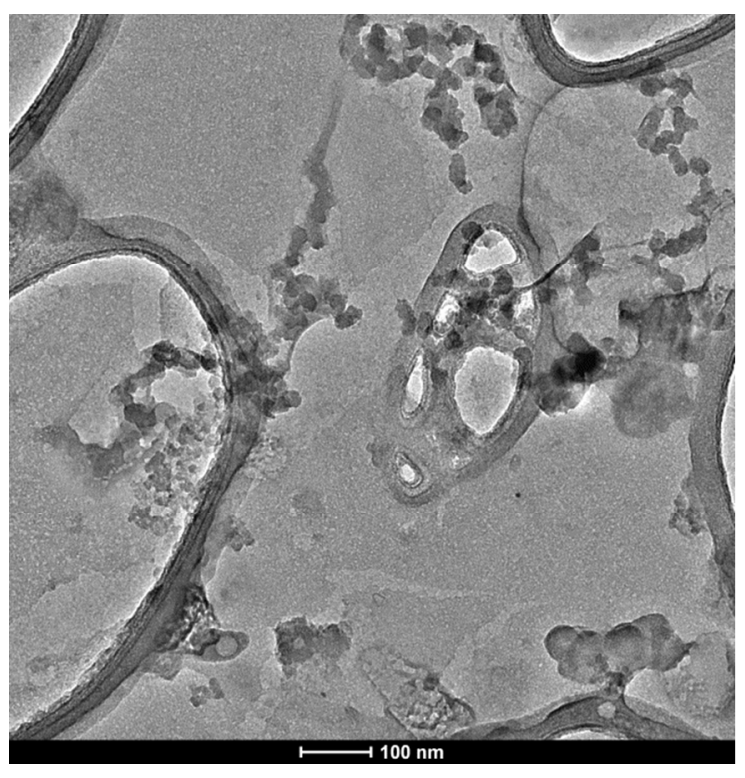

Figure S5. A sample pre-dissolved with no acetic acid present. Large clusters of nanoparticles, consistent with DLS, are present in the solution and deposited onto the grid. 


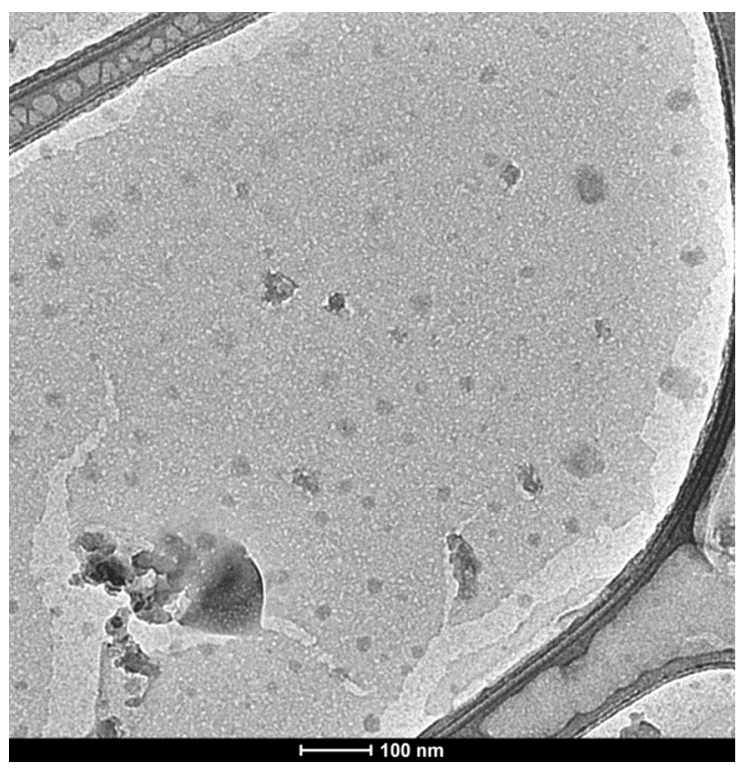

Figure S6. A sample pre-dissolved with acetic acid. In comparison to Figure S4 and consistent with the DLS measurements (main paper), the presence of nanoparticles is ambiguous - the size and nuclearity of the species that appear on the grid are smaller than those without acetic acid. 
4.2 SEM and EDX analysis of electrodes used with $\mathbf{3}$

SEM-EDX measurements were taken using a Hitachi SU-70 analytical scanning microscope.

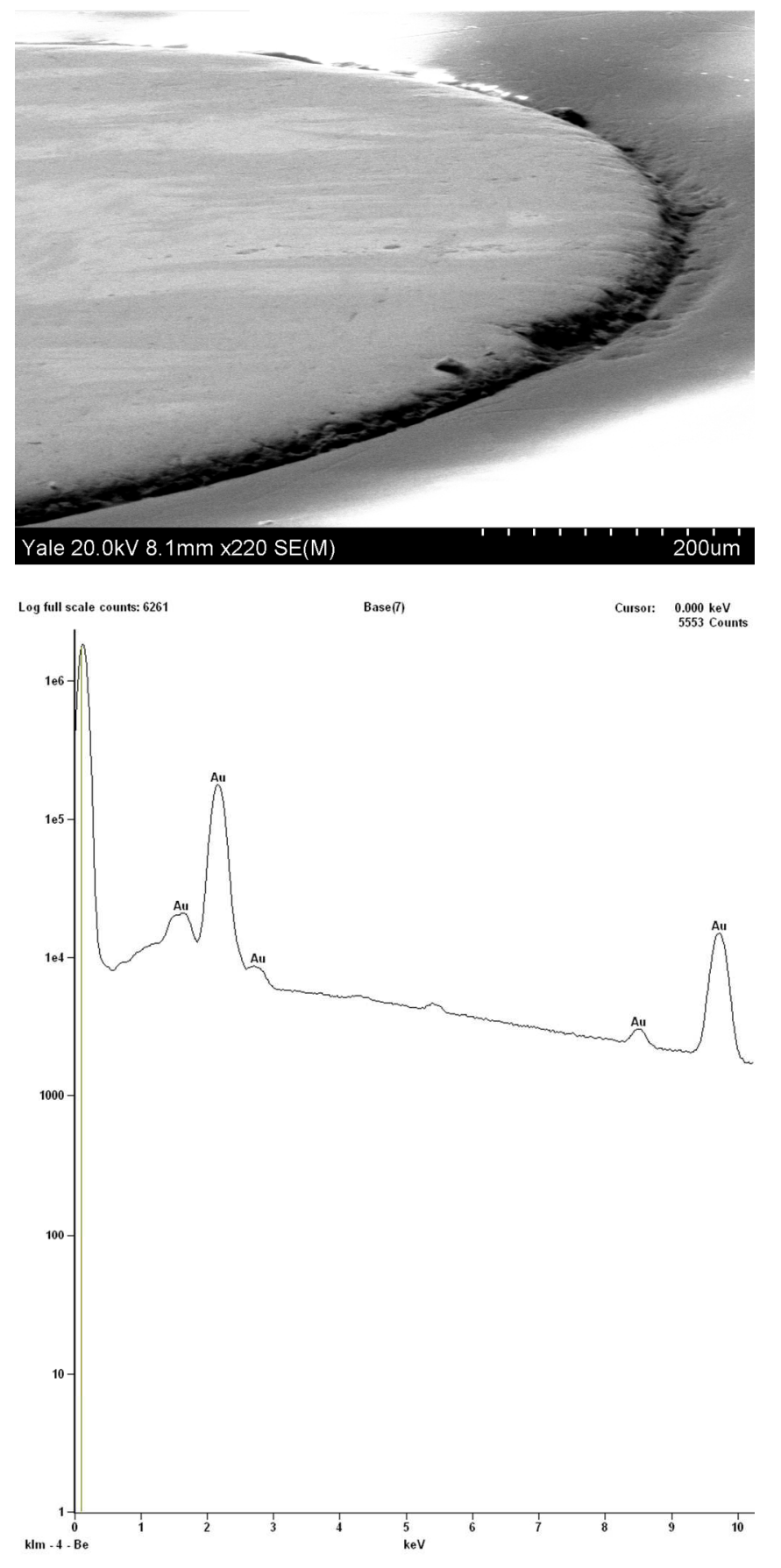

Figure S7. SEM-EDX of unpolished gold electrode used for electrochemical oxygen assay of 1 $\mathrm{mM} 2$ 'e in $0.25 \mathrm{M} \mathrm{Na}_{2} \mathrm{SO}_{4}$ and $100 \mathrm{mM}$ acetate buffer. Measurements show no traces of iridium on the electrode surface. 


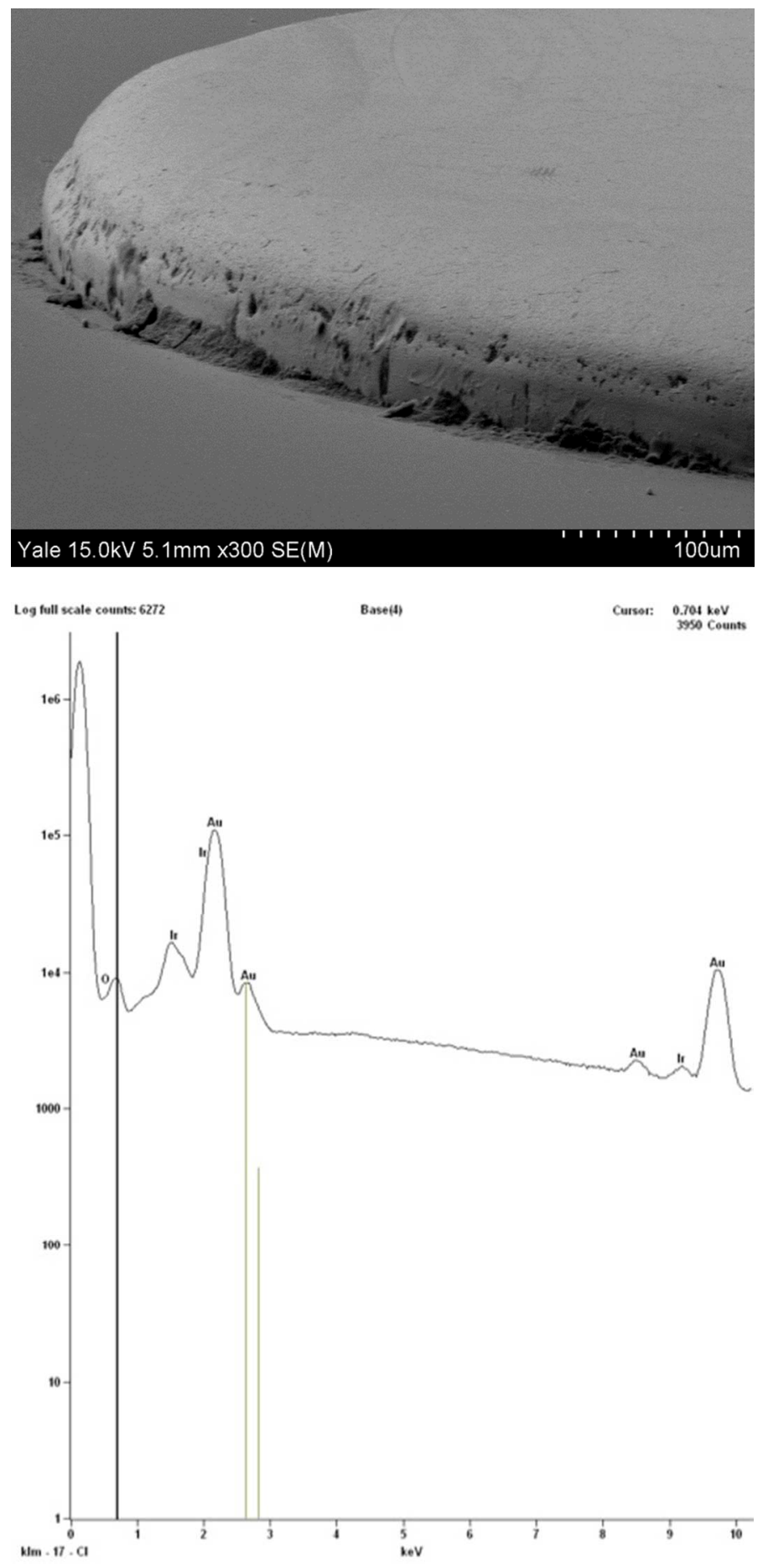

Figure S8. SEM-EDX of unpolished gold electrode used for electrochemical oxygen assay of 1 mM 2'e in $0.25 \mathrm{M} \mathrm{Na}_{2} \mathrm{SO}_{4}$. While no iridium deposits could be visually observed by SEM, the EDX analysis showed small traces of iridium deposited on the electrode. Supporting the data collected in the DLS studies, $\mathbf{2}$ 'e forms small nanoparticles in the absence of acetate ligands. 


\section{Long-term oxygen assay with 3 and $\mathrm{NaIO}_{4}$}

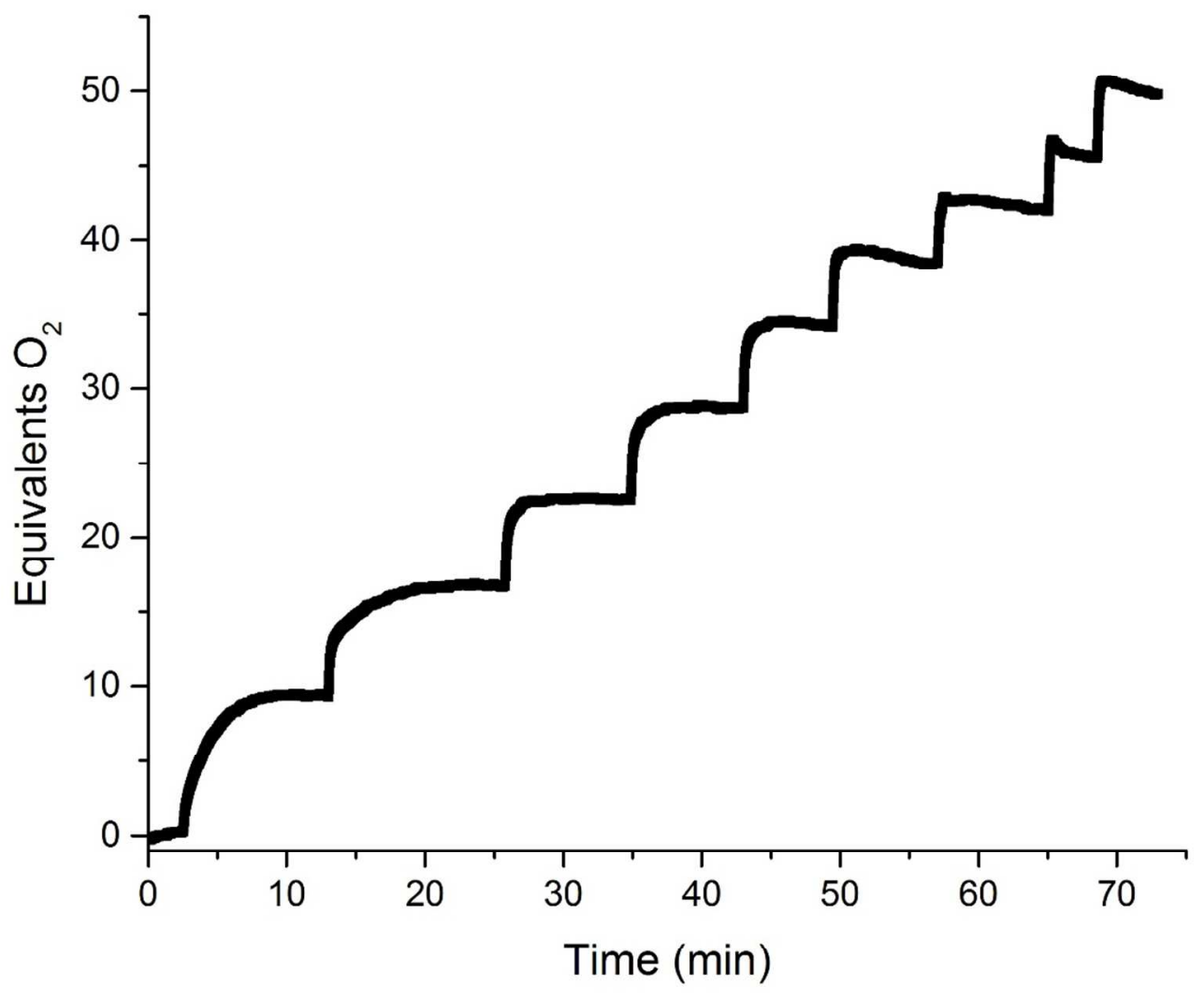

Figure S9. $\mathrm{O}_{2}$ production of $1 \mu \mathrm{M} 3$ and 200 equivalents of acetic acid during multiple additions of 25 equivalents of $\mathrm{NaIO}_{4}$ in $\mathrm{H}_{2} \mathrm{O}$ as measured by Clark-type electrode in liquid phase. Rate decrease is due to successive dilution from oxidant injection; slight decrease in $\mathrm{O}_{2}$ concentrations after injection are due to $\mathrm{O}_{2}$ bubbles forming from the dissolved $\mathrm{O}_{2}$ gas in the over-saturated water solution. 


\section{Electrochemical characterization of 3}
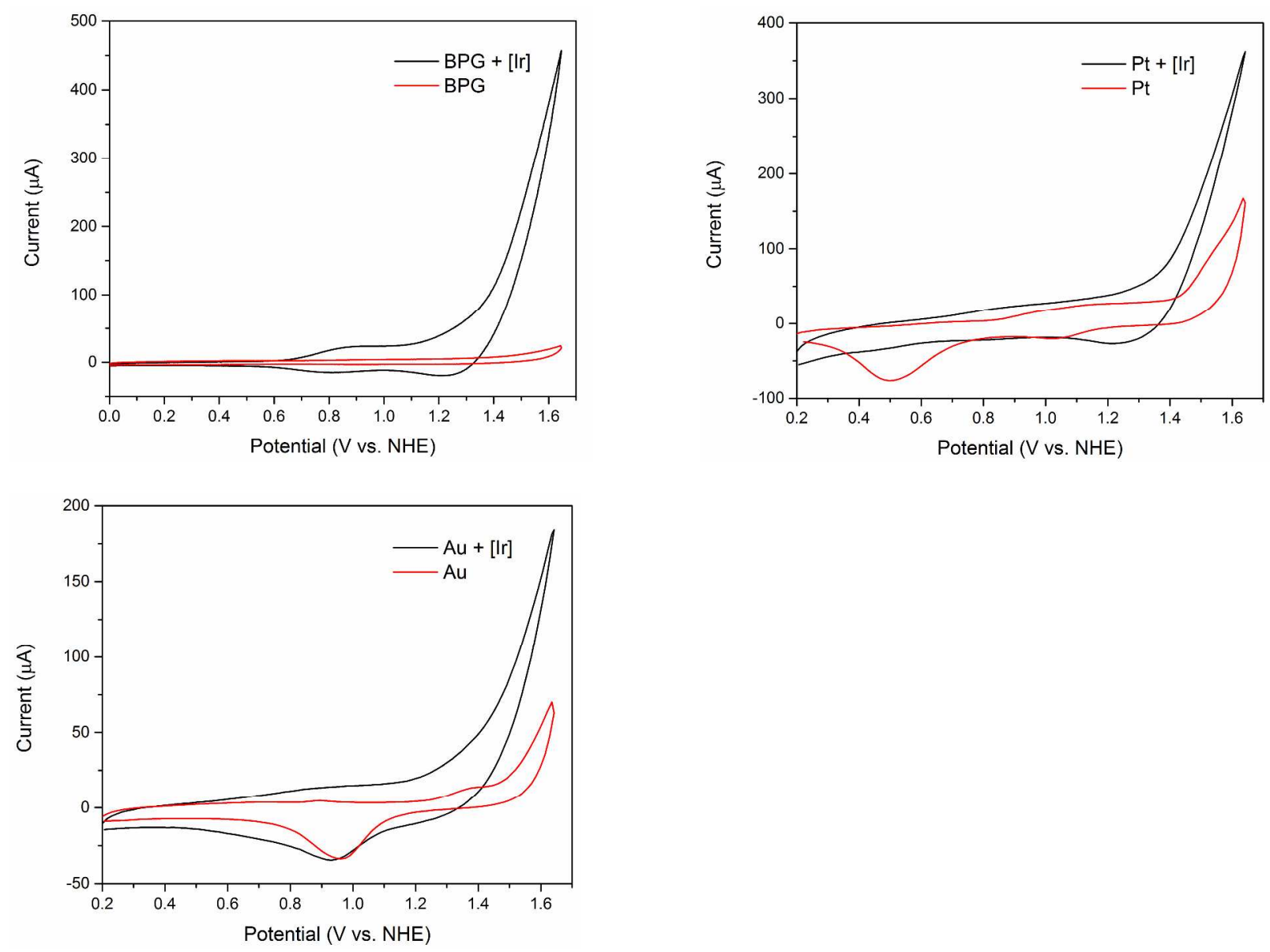

Figure S10. Cyclic voltammograms of $1 \mathrm{mM} 3$ (black lines) in $0.25 \mathrm{M} \mathrm{Na}_{2} \mathrm{SO}_{4}$ at (top left) homemade basal plane graphite (BPG) electrode, ${ }^{3}$ (top right) a platinum electrode, (bottom) a gold electrode. CVs of electrodes in blank electrolyte were taken under the same setup (red lines). Experiments were carried out at $\mathrm{pH} 2.75$ (adjusted with $\mathrm{H}_{2} \mathrm{SO}_{4}$ ) with an $\mathrm{Ag} / \mathrm{AgCl}$ electrode, Pt wire counter electrode, and $500 \mathrm{mV} / \mathrm{s}$ scan rate.

\footnotetext{
${ }^{3}$ Cady, C. W.; Shinopoulos, K. E.; Crabtree, R. H.; Brudvig, G. W. Dalt. Trans. 2010, 39 (16), 3985-3989.
} 

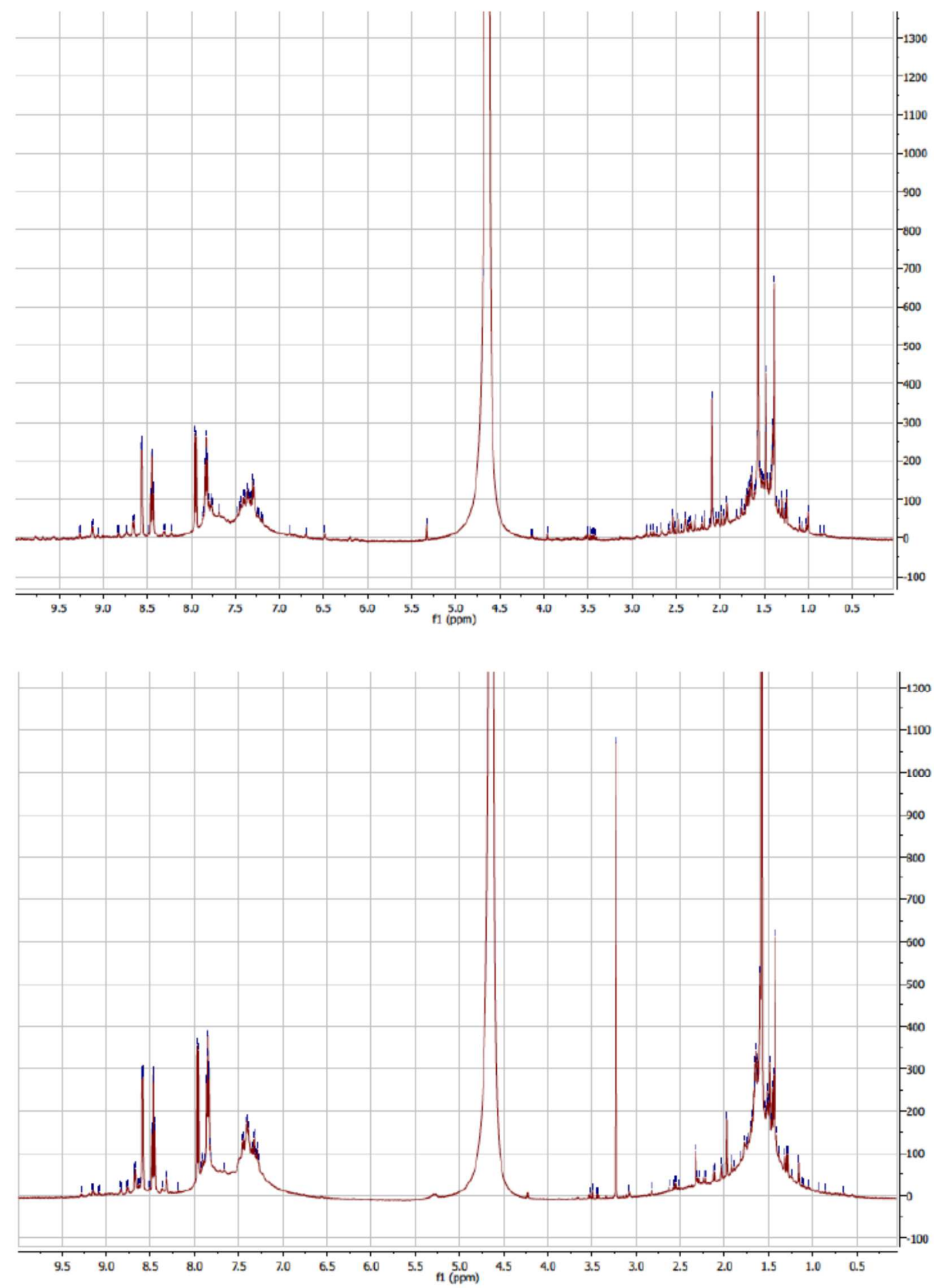

Figure S11. ${ }^{1} \mathrm{H}$ NMR spectra of electrochemically activated $1 \mathrm{mM} 3$ in $0.25 \mathrm{M} \mathrm{Na}_{2} \mathrm{SO}_{4}(8$ hours of bulk electrolysis at $1.0 \mathrm{~V}$ vs. NHE) at a platinum mesh electrode (top) and at a reticulated vitreous carbon electrode (bottom). Solutions were dried and redissolved in $\mathrm{D}_{2} \mathrm{O}$. The similarity between the spectra suggests that the electrode material is irrelevant to the activation process for 3. 


\section{7. $\quad{ }^{1} \mathrm{H}$ NMR spectra of $2,2^{\prime}$, and $2^{\prime} \mathrm{e}$}

These peaks covered a wide range, 9.5 to $6.0 \mathrm{ppm}$, with no dominant isomer being apparent. Unfortunately, peaks in the aliphatic region could not be resolved due to the signals from acetic acid and other organic by-products from $\mathrm{Cp}^{*}$ degradation. The peaks surrounding the acetic acid signal at $2.09 \mathrm{ppm}$ are likely from $\mathrm{AcO}$ adducts binding to $\mathrm{Ir}^{\mathrm{IV}}$ in different orientations; this would align well with what is observed in Figure 5 and with the shift in $\lambda_{\max }$ of $2^{\prime}$ upon addition of iodate and acetic acid. These adduct isomer signals also overwhelm the signals from the methyls of the pyalc ligand, further complicating interpretations of how many unique isomers exist in solution. It is unlikely that small metallic NPs are interfering with the signals, as previous DLS and TEM measurements (main paper, Figures S3-S5) have ruled out NP formation under the given conditions.

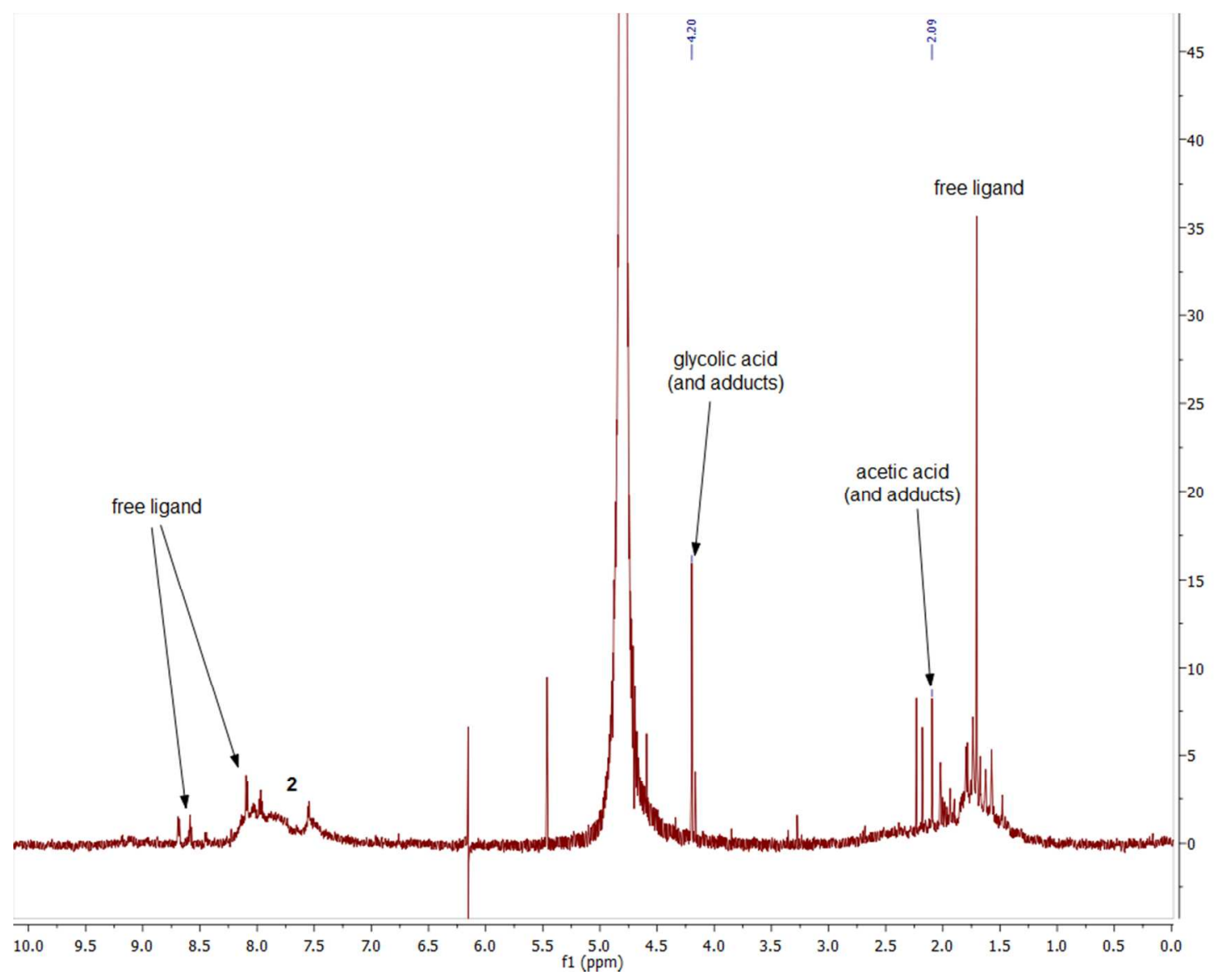

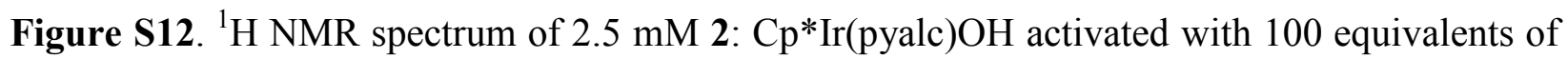
$\mathrm{NaIO}_{4}$ in $\mathrm{D}_{2} \mathrm{O}, \mathrm{pH}$ adjusted to 2.5 with $\mathrm{HNO}_{3}$. 


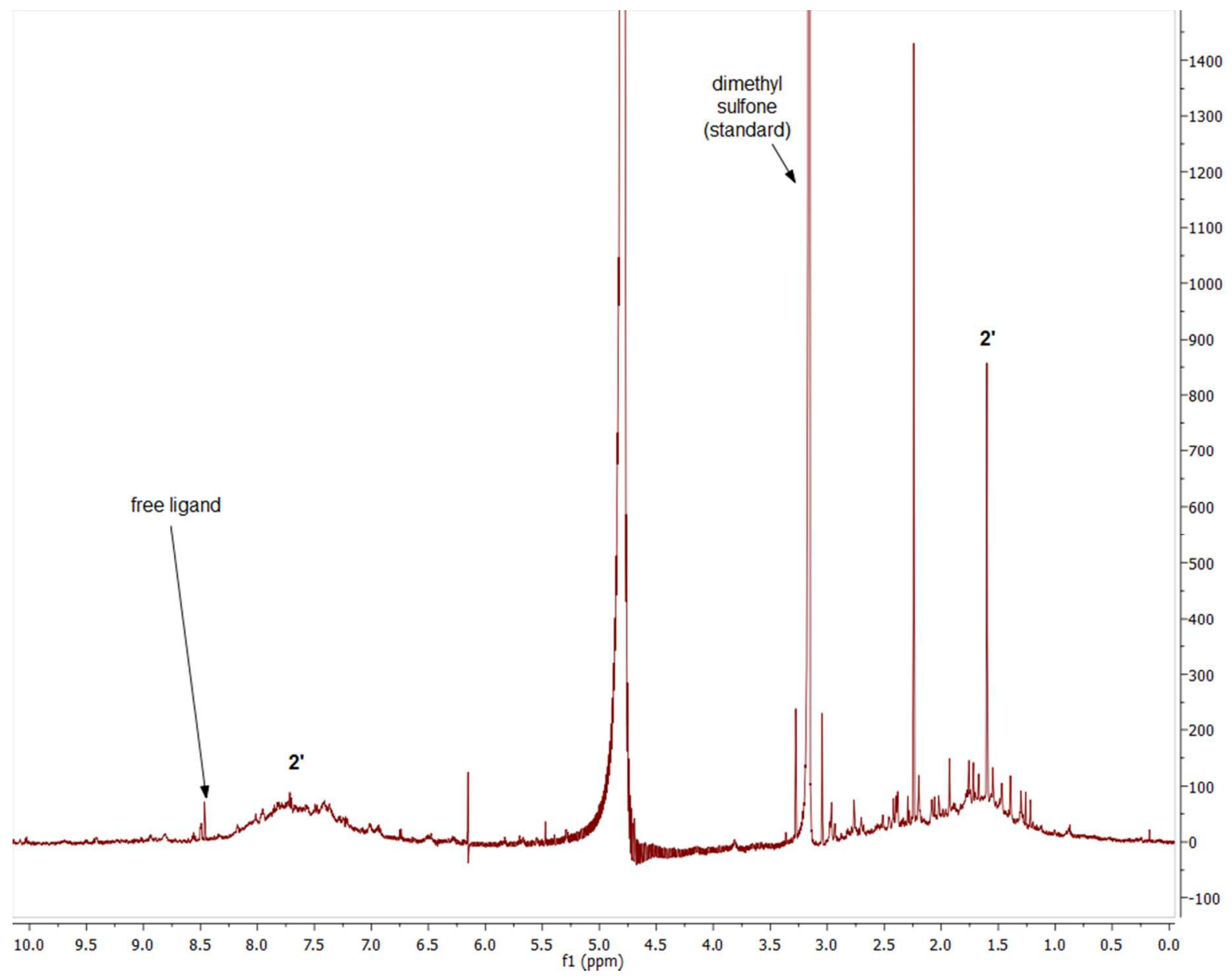

Figure S13. ${ }^{1} \mathrm{H}$ NMR spectrum of $2^{\prime}: 1.8 \mathrm{mM} 3$ activated by 10 equivalents of $\mathrm{NaIO}_{4}$ in $\mathrm{D}_{2} \mathrm{O}$,. The peak at $3.0 \mathrm{ppm}$ is a dimethylsulfone standard. Solution was dried and redissolved in $\mathrm{D}_{2} \mathrm{O}$. (Figure 9 in the main paper). 


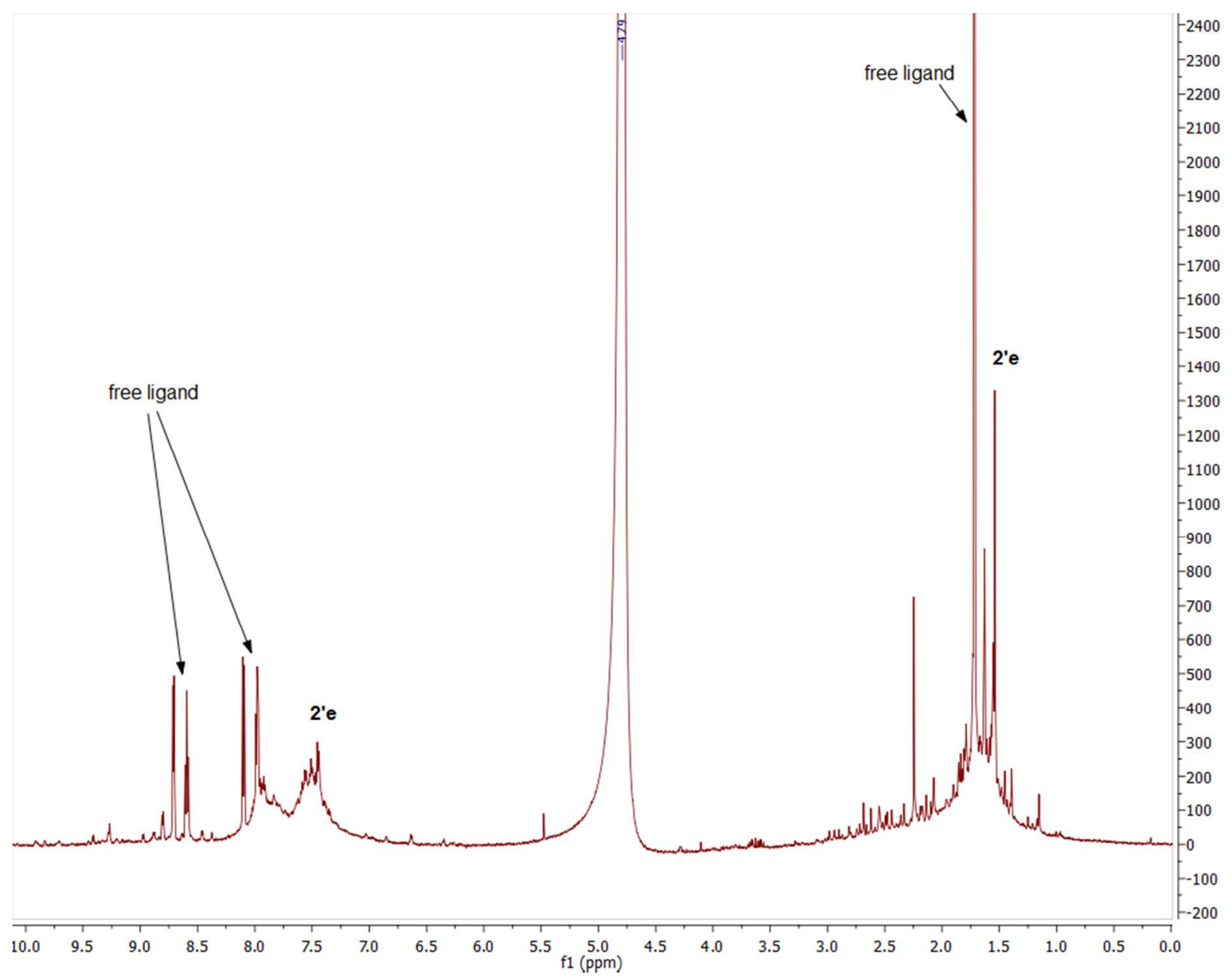

Figure S14. $1.8 \mathrm{mM} 3$ activated by bulk electrolysis (2'e) with a Pt electrode at $1.0 \mathrm{~V}$ over 8 hours in $\mathrm{Na}_{2} \mathrm{SO}_{4}$. Solution was dried and redissolved in $\mathrm{D}_{2} \mathrm{O}$. 


\section{DOSY of chemically activated $3\left(2^{\prime}\right)$}

A few peaks can be clearly seen in the DOSY spectrum, which would support our working hypothesis that many isomers of the active species exist in solution. Most of the species have a diffusion coefficient of about $4 \times 10^{-10} \mathrm{~m}^{2} \mathrm{~s}^{-1}$, although two are centered closer to $9 \times 10^{-10} \mathrm{~m}^{2} \mathrm{~s}^{-1}$. However, the signals are weak and overlapping which complicates determining an accurate diffusion coefficient as well as distinguishing a single complex. Additionally, there are many more peaks in the proton spectrum (Figure S13) which are not resolved in the DOSY spectrum.

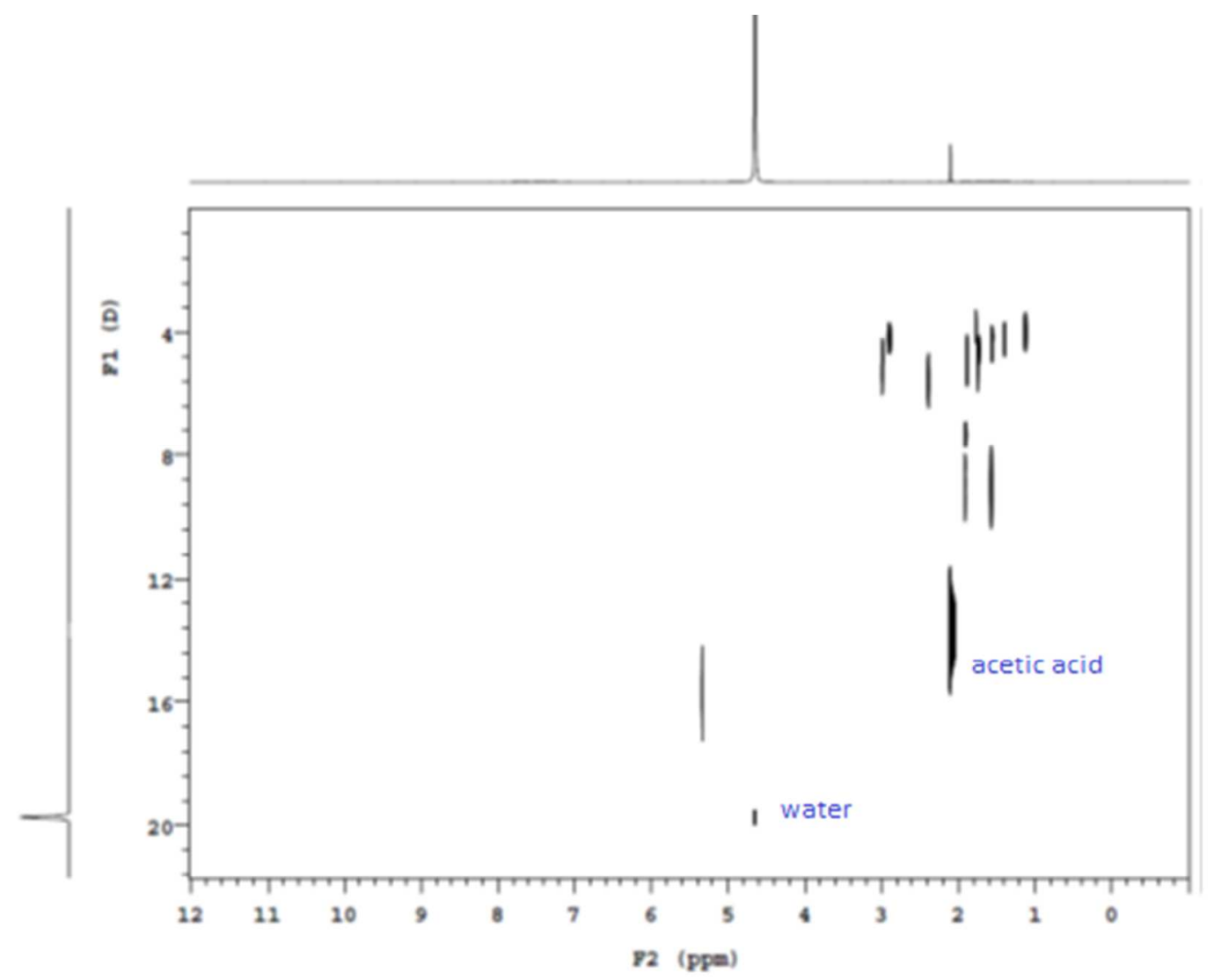

Figure S15. DOSY NMR spectrum of $2.5 \mathrm{mM} 3$ with 10 equivalents of $\mathrm{NaIO}_{4}$ and 150 equivalents of acetic acid- $\mathrm{d}_{4}$ in $\mathrm{D}_{2} \mathrm{O}$ at $25^{\circ} \mathrm{C}$. A more detailed ${ }^{1} \mathrm{H}$ proton spectrum is represented by Figure S13. The peak at $5.5 \mathrm{ppm}$ is an artifact from phasing and baseline correction.

Nevertheless, using the Stokes-Einstein equation (Equation 1), we can estimate the general size of the two types of species.

$$
R_{H}=\frac{k_{B} T}{6 \pi \eta D}
$$

$\mathrm{R}_{\mathrm{H}}$ is the Stokes radius, $\mathrm{k}_{\mathrm{B}}$ the Boltzmann constant, $T$ is temperature, $\eta$ is the viscosity constant of the solvent $\left(8.90 \times 10^{-4} \mathrm{~Pa} \cdot \mathrm{s}\right.$ for water at $\left.25^{\circ} \mathrm{C}\right)$, and $\mathrm{D}$ is the diffusion coefficient. For the species with a diffusion coefficient of $4 \times 10^{-10} \mathrm{~m}^{2} \mathrm{~s}^{-1}$, we can estimate that they have an 
approximate Stokes radius of $6 \AA$. For the species with a diffusion coefficient of $9 \times 10^{-10} \mathrm{~m}^{2} \mathrm{~s}^{-1}$, we can estimate that they have an approximate Stokes radius of $3 \AA$. These sizes are much smaller than expected for bimetallic species, given the crystal structure of the precursor; a radius of $6 \AA$ gives a volume roughly similar to 3 alone.

We hypothesize that the missing peaks from the DOSY that are visualized in the proton spectrum (Figure S13) represent the polymetallic species, which relax much more quickly than the monomeric Ir active species. If a metallic complex has some degree of paramagnetism, it may relax more quickly than it diffuses through the solution and thus DOSY measurements would be rendered useless. Previous DOSY measurements done on metallic complexes have mostly focused on alkali and alkaline metals, which do not have this issue. ${ }^{4}$ Despite this drawback, the DOSY measurements have indicated that monomeric species also likely exist in solution with the polymetallic species previously characterized by spectrometry and X-ray measurements, ${ }^{5}$ and we can now qualitatively assign which peaks from the monomeric and polynuclear species.

\footnotetext{
4 (a) Armstrong, D. R.; Kennedy, A. R.; Mulvey, R. E.; Robertson, S. D. Dalt. Trans. 2013, 42 (10), 3704-3711, (b) Li, D.; Keresztes, I.; Hopson, R.; Williard, P. G. Acc. Chem. Res. 2009, 42 (2), 270-280.

${ }^{5}$ Hintermair, U.; Sheehan, S. W.; Parent, A. R.; Ess, D. H.; Richens, D. T.; Vaccaro, P. H.; Brudvig, G. W.; Crabtree, R. H. J. Am. Chem. Soc. 2013, 135 (29), 10837-10851.
} 


\section{X-ray crystal structure and data for 3}

Without a co-crystallization agent, $\mathbf{3}$ forms small, poor-quality yellow crystals which are twinned and often cracked. Likely, each unit of $\mathbf{3}$ stacks antiplanar on top of one another in a random matter (Figure S16), which has been documented in previous $\left[\operatorname{Ir}^{\mathrm{I}}(\mathrm{CO})_{2}\right.$ (chelate)] systems. ${ }^{6}$ Below is the data collected from $\mathbf{3}$ by itself, as a comparison to the data collected of $\mathbf{3}$ with a cocrystallization agent (S24).

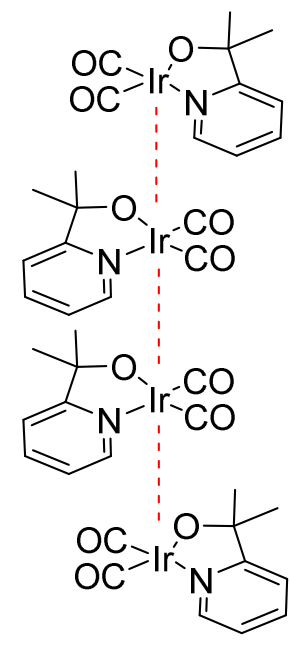

Figure S16. $\operatorname{Ir}(\mathrm{CO})_{2}($ pyalc) moieties stacking antiplanar to one another in random fashion.

\section{Crystallographic Data}

Low-temperature diffraction data ( $\omega$-scans) were collected on a Rigaku MicroMax-007HF diffractometer coupled to a Saturn994+ CCD detector with $\mathrm{Cu} \mathrm{K \alpha}(\lambda=1.54178 \AA)$ for structure 3. The structure was solved by direct methods using SHELXT and was refined against $F^{2}$ on all data by full-matrix least squares with SHELXL (Sheldrick, G. M. Acta Cryst. 2008, A64, 112122). All non-hydrogen atoms were refined anisotropically. The hydrogen atoms were included in the model at geometrically calculated positions and refined using a riding model. The isotropic displacement parameters of all hydrogen atoms were fixed to 1.2 times the $U$ value of the atoms to which they are linked (1.5 times for methyl groups). CCDC 1403936 contains the supplementary crystallographic data for this paper. The data can be obtained free of charge from The Cambridge Crystallographic Data Centre. Full details of the X-ray structure determination are in the CIF included as Supporting Information.

\footnotetext{
${ }^{6}$ Trzaska, S. T.; Swager, T. M. Chem. Mater. 1998, 10 (1), 438-443.
} 


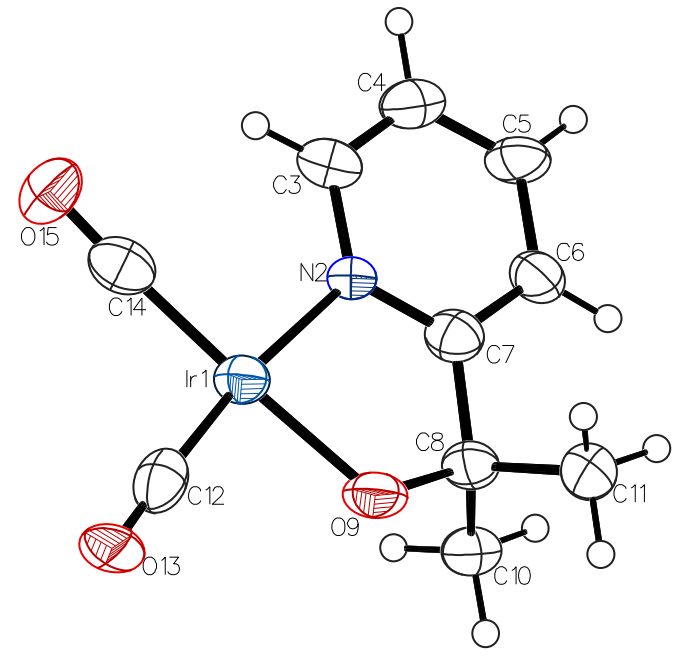

Figure S17. Crystal structure with full numbering scheme of 3. All atoms shown are depicted with $50 \%$ thermal contours. The hydrogen atoms are shown as spheres.

Table S1. Crystal data and structure refinement for 3 .

Empirical formula

Formula weight

Temperature

Wavelength

Crystal system

Space group

Unit cell dimensions

Volume

Z

Density (calculated)

Absorption coefficient

$\mathrm{F}(000)$

Crystal size

Crystal color and habit

Diffractometer

$\theta$ range for data collection

Index ranges

Reflections collected
$\mathrm{C}_{10} \mathrm{H}_{10} \mathrm{IrNO}_{3}$

384.39

93(2) K

$1.54187 \AA$

Monoclinic

$P 21 / n$

$\mathrm{a}=8.0620(2) \AA$

$\alpha=90^{\circ}$.

$\mathrm{b}=14.5888(3) \AA$

$\beta=100.895(7)^{\circ}$.

$\mathrm{c}=9.2740(7) \AA$

1071.10(9) $\AA^{3}$

4

$2.384 \mathrm{Mg} / \mathrm{m}^{3}$

$24.047 \mathrm{~mm}^{-1}$

712

$0.180 \times 0.140 \times 0.040 \mathrm{~mm}^{3}$

Colorless Plate

Rigaku Saturn 944+ CCD

5.727 to $68.163^{\circ}$.

$-9 \leq h \leq 9,-17 \leq k \leq 17,-11 l \leq 11$

34892 
Independent reflections

Observed reflections $(\mathrm{I}>2 \sigma(\mathrm{I}))$

Completeness to $\theta=67.687^{\circ}$

Absorption correction

Max. and min. transmission

Solution method

Refinement method

Data / restraints / parameters

Goodness-of-fit on $\mathrm{F}^{2}$

Final $\mathrm{R}$ indices $[\mathrm{I}>2 \sigma(\mathrm{I})]$

$\mathrm{R}$ indices (all data)

Largest diff. peak and hole
$1945[\mathrm{R}(\mathrm{int})=0.1389]$

1658

$99.6 \%$

Semi-empirical from equivalents

1.000 and 0.604

SHELXS-2013 (Sheldrick, 2013)

SHELXL-2014/7 (Sheldrick, 2014)

1945 / 0 / 138

1.148

$\mathrm{R} 1=0.0686, \mathrm{wR} 2=0.1927$

$\mathrm{R} 1=0.0752, \mathrm{wR} 2=0.2066$

2.380 and -4.196 e. $\AA^{-3}$

Table S2. Atomic coordinates ( $\left.\mathrm{x} 10^{4}\right)$ and equivalent isotropic displacement parameters $\left(\AA^{2} \mathrm{x}\right.$ $\left.10^{3}\right)$ for 3. $\mathrm{U}(\mathrm{eq})$ is defined as one third of the trace of the orthogonalized $\mathrm{U}^{\mathrm{ij}}$ tensor.

\begin{tabular}{lllll}
\hline & & & & \\
& & & & \\
& & & & \\
& & & & \\
$\mathrm{Ir}(1)$ & $6537(1)$ & $4295(1)$ & $3082(1)$ & $37(1)$ \\
$\mathrm{N}(2)$ & $4804(10)$ & $3750(6)$ & $4266(10)$ & $33(2)$ \\
$\mathrm{C}(3)$ & $3135(14)$ & $3975(9)$ & $4086(12)$ & $38(2)$ \\
$\mathrm{C}(4)$ & $2109(16)$ & $3520(9)$ & $4840(14)$ & $46(3)$ \\
$\mathrm{C}(5)$ & $2738(14)$ & $2870(9)$ & $5918(14)$ & $44(3)$ \\
$\mathrm{C}(6)$ & $4436(15)$ & $2668(9)$ & $6115(14)$ & $46(3)$ \\
$\mathrm{C}(7)$ & $5470(14)$ & $3103(8)$ & $5265(13)$ & $40(3)$ \\
$\mathrm{C}(8)$ & $7361(13)$ & $2910(8)$ & $5425(14)$ & $40(2)$ \\
$\mathrm{O}(9)$ & $8019(9)$ & $3414(6)$ & $4337(9)$ & $47(2)$ \\
$\mathrm{C}(10)$ & $8270(15)$ & $3180(10)$ & $6943(13)$ & $44(3)$ \\
$\mathrm{C}(11)$ & $7595(16)$ & $1880(9)$ & $5100(16)$ & $50(3)$ \\
$\mathrm{C}(12)$ & $8229(19)$ & $4659(9)$ & $2125(14)$ & $48(3)$ \\
$\mathrm{O}(13)$ & $9348(10)$ & $4873(6)$ & $1545(10)$ & $53(2)$ \\
$\mathrm{C}(14)$ & $5064(16)$ & $5093(9)$ & $1933(14)$ & $48(3)$ \\
$\mathrm{O}(15)$ & $4098(13)$ & $5558(6)$ & $1192(11)$ & $53(2)$ \\
& & & & \\
\hline
\end{tabular}


Table S3. Bond lengths $[\AA]$ and angles $\left[{ }^{\circ}\right]$ for 3 .

\begin{tabular}{|c|c|}
\hline $\operatorname{Ir}(1)-C(12)$ & $1.841(16)$ \\
\hline $\operatorname{Ir}(1)-C(14)$ & $1.850(14)$ \\
\hline $\operatorname{Ir}(1)-\mathrm{O}(9)$ & $1.978(8)$ \\
\hline $\operatorname{Ir}(1)-\mathrm{N}(2)$ & $2.090(8)$ \\
\hline $\mathrm{N}(2)-\mathrm{C}(7)$ & $1.360(14)$ \\
\hline $\mathrm{N}(2)-\mathrm{C}(3)$ & $1.364(14)$ \\
\hline$C(3)-C(4)$ & $1.353(18)$ \\
\hline $\mathrm{C}(3)-\mathrm{H}(3)$ & 0.9500 \\
\hline$C(4)-C(5)$ & $1.401(18)$ \\
\hline $\mathrm{C}(4)-\mathrm{H}(4)$ & 0.9500 \\
\hline$C(5)-C(6)$ & $1.379(16)$ \\
\hline $\mathrm{C}(5)-\mathrm{H}(5)$ & 0.9500 \\
\hline$C(6)-C(7)$ & $1.402(17)$ \\
\hline $\mathrm{C}(6)-\mathrm{H}(6)$ & 0.9500 \\
\hline$C(7)-C(8)$ & $1.529(15)$ \\
\hline $\mathrm{C}(8)-\mathrm{O}(9)$ & $1.428(14)$ \\
\hline $\mathrm{C}(8)-\mathrm{C}(10)$ & $1.511(16)$ \\
\hline $\mathrm{C}(8)-\mathrm{C}(11)$ & $1.551(17)$ \\
\hline $\mathrm{C}(10)-\mathrm{H}(10 \mathrm{~A})$ & 0.9800 \\
\hline $\mathrm{C}(10)-\mathrm{H}(10 \mathrm{~B})$ & 0.9800 \\
\hline $\mathrm{C}(10)-\mathrm{H}(10 \mathrm{C})$ & 0.9800 \\
\hline $\mathrm{C}(11)-\mathrm{H}(11 \mathrm{~A})$ & 0.9800 \\
\hline $\mathrm{C}(11)-\mathrm{H}(11 \mathrm{~B})$ & 0.9800 \\
\hline $\mathrm{C}(11)-\mathrm{H}(11 \mathrm{C})$ & 0.9800 \\
\hline $\mathrm{C}(12)-\mathrm{O}(13)$ & $1.176(17)$ \\
\hline $\mathrm{C}(14)-\mathrm{O}(15)$ & $1.156(16)$ \\
\hline$C(12)-\operatorname{Ir}(1)-C(14)$ & $89.7(5)$ \\
\hline $\mathrm{C}(12)-\operatorname{Ir}(1)-\mathrm{O}(9)$ & $92.9(4)$ \\
\hline $\mathrm{C}(14)-\operatorname{Ir}(1)-\mathrm{O}(9)$ & $177.3(4)$ \\
\hline $\mathrm{C}(12)-\operatorname{Ir}(1)-\mathrm{N}(2)$ & $173.1(5)$ \\
\hline $\mathrm{C}(14)-\operatorname{Ir}(1)-\mathrm{N}(2)$ & $97.0(4)$ \\
\hline
\end{tabular}




$\begin{array}{ll}\mathrm{O}(9)-\mathrm{Ir}(1)-\mathrm{N}(2) & 80.4(3) \\ \mathrm{C}(7)-\mathrm{N}(2)-\mathrm{C}(3) & 120.3(10) \\ \mathrm{C}(7)-\mathrm{N}(2)-\mathrm{Ir}(1) & 113.8(7) \\ \mathrm{C}(3)-\mathrm{N}(2)-\mathrm{Ir}(1) & 125.9(8) \\ \mathrm{C}(4)-\mathrm{C}(3)-\mathrm{N}(2) & 120.2(12) \\ \mathrm{C}(4)-\mathrm{C}(3)-\mathrm{H}(3) & 119.9 \\ \mathrm{~N}(2)-\mathrm{C}(3)-\mathrm{H}(3) & 119.9 \\ \mathrm{C}(3)-\mathrm{C}(4)-\mathrm{C}(5) & 121.8(12) \\ \mathrm{C}(3)-\mathrm{C}(4)-\mathrm{H}(4) & 119.1 \\ \mathrm{C}(5)-\mathrm{C}(4)-\mathrm{H}(4) & 119.1 \\ \mathrm{C}(6)-\mathrm{C}(5)-\mathrm{C}(4) & 117.1(11) \\ \mathrm{C}(6)-\mathrm{C}(5)-\mathrm{H}(5) & 121.5 \\ \mathrm{C}(4)-\mathrm{C}(5)-\mathrm{H}(5) & 121.5 \\ \mathrm{C}(5)-\mathrm{C}(6)-\mathrm{C}(7) & 120.7(11) \\ \mathrm{C}(5)-\mathrm{C}(6)-\mathrm{H}(6) & 119.7 \\ \mathrm{C}(7)-\mathrm{C}(6)-\mathrm{H}(6) & 119.7 \\ \mathrm{~N}(2)-\mathrm{C}(7)-\mathrm{C}(6) & 119.8(10) \\ \mathrm{N}(2)-\mathrm{C}(7)-\mathrm{C}(8) & 116.9(10) \\ \mathrm{C}(6)-\mathrm{C}(7)-\mathrm{C}(8) & 123.4(10) \\ \mathrm{O}(9)-\mathrm{C}(8)-\mathrm{C}(10) & 110.1(9) \\ \mathrm{O}(9)-\mathrm{C}(8)-\mathrm{C}(7) & 109.5(9) \\ \mathrm{C}(10)-\mathrm{C}(8)-\mathrm{C}(7) & 110.0(9) \\ \mathrm{O}(9)-\mathrm{C}(8)-\mathrm{C}(11) & 106.6(9) \\ \mathrm{C}(10)-\mathrm{C}(8)-\mathrm{C}(11) & 112.2(11) \\ \mathrm{C}(7)-\mathrm{C}(8)-\mathrm{C}(11) & 108.3(9) \\ \mathrm{C}(8)-\mathrm{O}(9)-\mathrm{Ir}(1) & 119.1(6) \\ \mathrm{C}(8)-\mathrm{C}(10)-\mathrm{H}(10 \mathrm{~A}) & 109.5 \\ \mathrm{C}(8)-\mathrm{C}(10)-\mathrm{H}(10 \mathrm{~B}) & 109.5 \\ \mathrm{H}(10 \mathrm{~A})-\mathrm{C}(10)-\mathrm{H}(10 \mathrm{~B}) & 109.5 \\ \mathrm{C}(8)-\mathrm{C}(10)-\mathrm{H}(10 \mathrm{C}) & 109.5 \\ \mathrm{H}(10 \mathrm{~A})-\mathrm{C}(10)-\mathrm{H}(10 \mathrm{C}) & 109.5 \\ \mathrm{H}(10 \mathrm{~B})-\mathrm{C}(10)-\mathrm{H}(10 \mathrm{C}) & 109.5 \\ \mathrm{C}(8)-\mathrm{C}(11)-\mathrm{H}(11 \mathrm{~A}) & 109.5 \\ \mathrm{H}(1)-\mathrm{C}(11)-\mathrm{H}(11 \mathrm{~B}) & 109.5 \\ \mathrm{C}(8)-\mathrm{C}(11)-\mathrm{H}(11 \mathrm{C}) & 109.5 \\ & \end{array}$


$\mathrm{H}(11 \mathrm{~A})-\mathrm{C}(11)-\mathrm{H}(11 \mathrm{C}) \quad 109.5$

$\mathrm{H}(11 \mathrm{~B})-\mathrm{C}(11)-\mathrm{H}(11 \mathrm{C}) \quad 109.5$

$\mathrm{O}(13)-\mathrm{C}(12)-\operatorname{Ir}(1) \quad 177.8(11)$

$\mathrm{O}(15)-\mathrm{C}(14)-\operatorname{Ir}(1) \quad 176.9(11)$

Symmetry transformations used to generate equivalent atoms:

Table S4. Anisotropic displacement parameters $\left(\AA^{2} \times 10^{3}\right)$ for 3. The anisotropic displacement factor exponent takes the form: $-2 p^{2}\left[h^{2} a^{* 2} U^{11}+\ldots+2 h k a^{*} b^{*} U^{12}\right]$

\begin{tabular}{lcccccc}
\hline & $\mathrm{U}^{11}$ & $\mathrm{U}^{22}$ & $\mathrm{U}^{33}$ & $\mathrm{U}^{23}$ & $\mathrm{U}^{13}$ & $\mathrm{U}^{12}$ \\
\hline $\mathrm{Ir}(1)$ & $30(1)$ & $42(1)$ & $38(1)$ & $1(1)$ & $6(1)$ & $-1(1)$ \\
$\mathrm{N}(2)$ & $23(4)$ & $33(4)$ & $41(5)$ & $3(4)$ & $3(4)$ & $-1(3)$ \\
$\mathrm{C}(3)$ & $32(6)$ & $52(6)$ & $33(6)$ & $-10(5)$ & $9(4)$ & $-5(5)$ \\
$\mathrm{C}(4)$ & $33(6)$ & $54(7)$ & $47(7)$ & $-4(6)$ & $-1(5)$ & $3(5)$ \\
$\mathrm{C}(5)$ & $26(5)$ & $61(7)$ & $47(7)$ & $4(5)$ & $12(5)$ & $3(5)$ \\
$\mathrm{C}(6)$ & $39(6)$ & $52(7)$ & $45(7)$ & $14(5)$ & $-1(5)$ & $-13(5)$ \\
$\mathrm{C}(7)$ & $38(6)$ & $46(6)$ & $35(6)$ & $-2(5)$ & $3(5)$ & $-4(5)$ \\
$\mathrm{C}(8)$ & $27(5)$ & $41(6)$ & $54(7)$ & $0(5)$ & $14(5)$ & $3(4)$ \\
$\mathrm{O}(9)$ & $26(4)$ & $63(5)$ & $52(5)$ & $9(4)$ & $5(3)$ & $-3(4)$ \\
$\mathrm{C}(10)$ & $32(6)$ & $50(7)$ & $47(7)$ & $3(5)$ & $0(5)$ & $-1(5)$ \\
$\mathrm{C}(11)$ & $40(7)$ & $44(7)$ & $65(9)$ & $9(6)$ & $11(6)$ & $0(5)$ \\
$\mathrm{C}(12)$ & $63(9)$ & $37(7)$ & $37(6)$ & $-2(5)$ & $-7(6)$ & $4(6)$ \\
$\mathrm{O}(13)$ & $26(4)$ & $69(6)$ & $68(6)$ & $18(5)$ & $21(4)$ & $-2(4)$ \\
$\mathrm{C}(14)$ & $41(6)$ & $57(7)$ & $48(7)$ & $-6(6)$ & $15(5)$ & $-7(6)$ \\
$\mathrm{O}(15)$ & $52(6)$ & $61(5)$ & $50(6)$ & $17(4)$ & $20(5)$ & $19(4)$ \\
& & & & & & \\
\hline
\end{tabular}


Table S5. Hydrogen coordinates ( $\left.\times 10^{4}\right)$ and isotropic displacement parameters $\left(\AA^{2} \times 10^{3}\right)$ for 3 .

\begin{tabular}{|c|c|c|c|c|}
\hline & $\mathrm{x}$ & $\mathrm{y}$ & $\mathrm{z}$ & $\mathrm{U}(\mathrm{eq})$ \\
\hline $\mathrm{H}(3)$ & 2691 & 4453 & 3430 & 46 \\
\hline $\mathrm{H}(4)$ & 932 & 3644 & 4632 & 55 \\
\hline $\mathrm{H}(5)$ & 2024 & 2581 & 6488 & 53 \\
\hline $\mathrm{H}(6)$ & 4911 & 2230 & 6833 & 55 \\
\hline $\mathrm{H}(10 \mathrm{~A})$ & 9472 & 3028 & 7052 & 66 \\
\hline $\mathrm{H}(10 \mathrm{~B})$ & 7784 & 2847 & 7680 & 66 \\
\hline $\mathrm{H}(10 \mathrm{C})$ & 8145 & 3841 & 7080 & 66 \\
\hline $\mathrm{H}(11 \mathrm{~A})$ & 7017 & 1740 & 4097 & 75 \\
\hline $\mathrm{H}(11 \mathrm{~B})$ & 7115 & 1507 & 5798 & 75 \\
\hline $\mathrm{H}(11 \mathrm{C})$ & 8802 & 1744 & 5201 & 75 \\
\hline
\end{tabular}


Table S6. Torsion angles $\left[{ }^{\circ}\right]$ for 3 .

\begin{tabular}{lc}
\hline $\mathrm{C}(7)-\mathrm{N}(2)-\mathrm{C}(3)-\mathrm{C}(4)$ & $-3.7(16)$ \\
$\mathrm{Ir}(1)-\mathrm{N}(2)-\mathrm{C}(3)-\mathrm{C}(4)$ & $174.6(8)$ \\
$\mathrm{N}(2)-\mathrm{C}(3)-\mathrm{C}(4)-\mathrm{C}(5)$ & $6.0(18)$ \\
$\mathrm{C}(3)-\mathrm{C}(4)-\mathrm{C}(5)-\mathrm{C}(6)$ & $-4.1(19)$ \\
$\mathrm{C}(4)-\mathrm{C}(5)-\mathrm{C}(6)-\mathrm{C}(7)$ & $0.1(19)$ \\
$\mathrm{C}(3)-\mathrm{N}(2)-\mathrm{C}(7)-\mathrm{C}(6)$ & $-0.3(16)$ \\
$\mathrm{Ir}(1)-\mathrm{N}(2)-\mathrm{C}(7)-\mathrm{C}(6)$ & $-178.8(9)$ \\
$\mathrm{C}(3)-\mathrm{N}(2)-\mathrm{C}(7)-\mathrm{C}(8)$ & $-178.4(10)$ \\
$\mathrm{Ir}(1)-\mathrm{N}(2)-\mathrm{C}(7)-\mathrm{C}(8)$ & $3.1(12)$ \\
$\mathrm{C}(5)-\mathrm{C}(6)-\mathrm{C}(7)-\mathrm{N}(2)$ & $2.0(19)$ \\
$\mathrm{C}(5)-\mathrm{C}(6)-\mathrm{C}(7)-\mathrm{C}(8)$ & $-179.9(12)$ \\
$\mathrm{N}(2)-\mathrm{C}(7)-\mathrm{C}(8)-\mathrm{O}(9)$ & $-5.9(14)$ \\
$\mathrm{C}(6)-\mathrm{C}(7)-\mathrm{C}(8)-\mathrm{O}(9)$ & $176.0(11)$ \\
$\mathrm{N}(2)-\mathrm{C}(7)-\mathrm{C}(8)-\mathrm{C}(10)$ & $115.3(11)$ \\
$\mathrm{C}(6)-\mathrm{C}(7)-\mathrm{C}(8)-\mathrm{C}(10)$ & $-62.8(15)$ \\
$\mathrm{N}(2)-\mathrm{C}(7)-\mathrm{C}(8)-\mathrm{C}(11)$ & $-121.8(11)$ \\
$\mathrm{C}(6)-\mathrm{C}(7)-\mathrm{C}(8)-\mathrm{C}(11)$ & $60.1(15)$ \\
$\mathrm{C}(10)-\mathrm{C}(8)-\mathrm{O}(9)-\operatorname{Ir}(1)$ & $-114.8(9)$ \\
$\mathrm{C}(7)-\mathrm{C}(8)-\mathrm{O}(9)-\operatorname{Ir}(1)$ & $6.2(12)$ \\
$\mathrm{C}(11)-\mathrm{C}(8)-\mathrm{O}(9)-\operatorname{Ir}(1)$ & $123.2(9)$ \\
\hline
\end{tabular}

Symmetry transformations used to generate equivalent atoms: 


\section{X-ray crystal structure and data for 3 co-crystallized with 1,4-butanediol}

Low-temperature diffraction data ( $\omega$-scans) were collected on a Rigaku MicroMax-007HF diffractometer coupled to a Saturn994+ CCD detector with $\mathrm{Cu} \mathrm{K \alpha}(\lambda=1.54178 \AA)$. The structure was solved by SHELXT and was refined against $\mathrm{F}^{2}$ on all data by full-matrix least squares with SHELXL (Sheldrick, G. M. Acta Cryst. 2008, A64, 112-122). All non-hydrogen atoms were refined anisotropically. Unless otherwise noted, hydrogen atoms were included in the model at geometrically calculated positions and refined using a riding model. The only exception is H16, which is freely refining. A hydrogen bond was identified between O16-H16...O9_\#4, where \#4 refers to the symmetry operation $\mathrm{x}, \mathrm{y}, \mathrm{z}-1$. The D...A distance was refined to a value of 2.743(3) $\AA$. The isotropic displacement parameters of all hydrogen atoms were fixed to 1.2 times the $\mathrm{U}$ value of the atoms to which they are linked (1.5 times for methyl groups). CCDC 1403937 contains the supplementary crystallographic data for this paper. The data can be obtained free of charge from The Cambridge Crystallographic Data Centre. Full details of the X-ray structure determination are in the CIF included as Supporting Information.

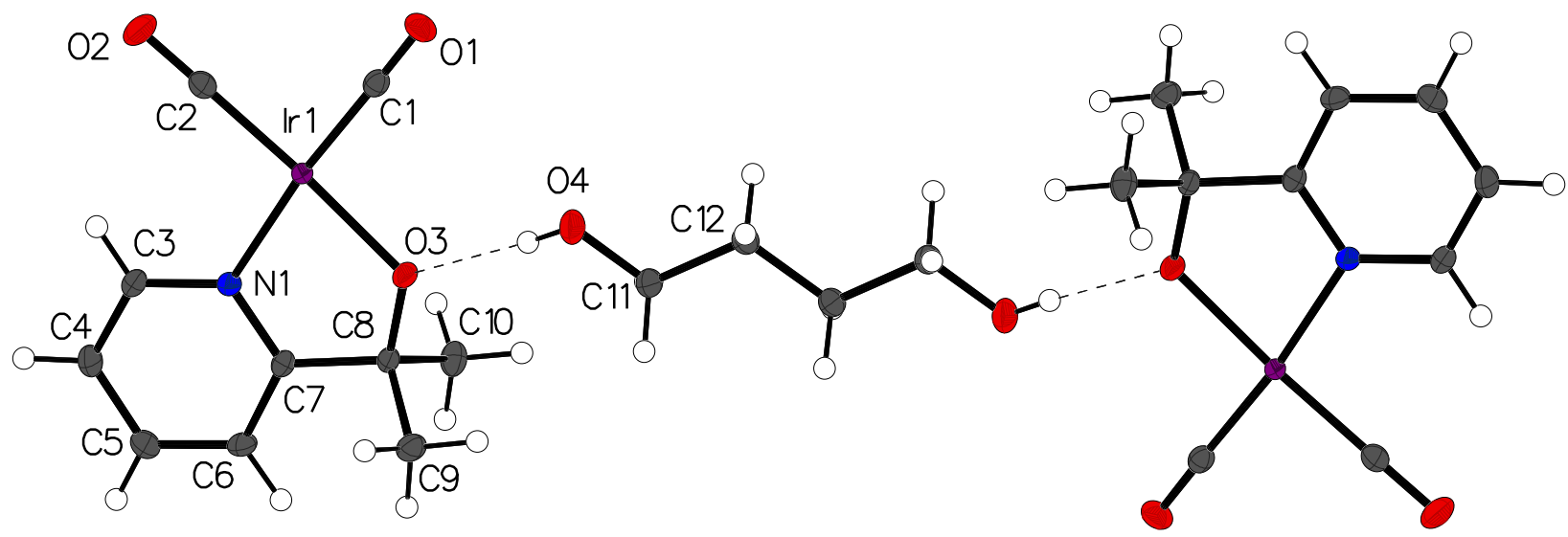

Figure S18. Crystal structure with full numbering scheme of 3 with 1,4-butanediol. All atoms shown are depicted with $50 \%$ thermal contours. The hydrogen atoms are shown as spheres.

Table S7. Crystal data and structure refinement for $\mathbf{3}+1$,4-butanediol

Empirical formula

Formula weight

Temperature

Wavelength

Crystal system

Space group

Unit cell dimensions
$\mathrm{C}_{12} \mathrm{H}_{15} \mathrm{IrNO}_{4}$

429.45

93(2) K

$0.71075 \AA$

Triclinic

$P \overline{1}$

$\mathrm{a}=8.2220(2) \AA \quad \mathrm{a}=97.700(7)^{\mathrm{o}}$

$\mathrm{b}=8.8397(3) \AA \quad \mathrm{b}=108.239(8)^{\circ}$ 
Volume

Z

Density (calculated)

Absorption coefficient

$\mathrm{F}(000)$

Crystal size

$\Theta$ range for data collection

Index ranges

Reflections collected

Independent reflections

Completeness to $\theta=25.242^{\circ}$

Absorption correction

Max. and min. transmission

Refinement method

Data / restraints / parameters

Goodness-of-fit on $\mathrm{F}^{2}$

Final $\mathrm{R}$ indices $[\mathrm{I}>2 \sigma(\mathrm{I})]$

$\mathrm{R}$ indices (all data)

Largest diff. peak and hole $\mathrm{c}=9.5097(7) \AA \quad \mathrm{g}=90.640(6)^{\mathrm{o}}$

$649.48(6) \AA^{3}$

2

$2.196 \mathrm{mg} / \mathrm{m}^{3}$

$10.284 \mathrm{~mm}^{-1}$

406

$0.200 \times 0.200 \times 0.050 \mathrm{~mm}^{3}$

3.014 to $27.470^{\circ}$.

$-10 \leq h \leq 10,-11 \leq k \leq 11,-12 \leq l \leq 12$

19760

$2985[\mathrm{R}(\mathrm{int})=0.0234]$

$99.8 \%$

Semi-empirical from equivalents

0.627 and 0.327

Full-matrix least-squares on $\mathrm{F}^{2}$

2985 / 0 / 169

1.180

$\mathrm{R} 1=0.0130, \mathrm{wR} 2=0.0308$

$\mathrm{R} 1=0.0135, \mathrm{wR} 2=0.0310$

0.983 and -0.608 e. $\AA^{-3}$ 
Table S8. Atomic coordinates $\left(\mathrm{x} 10^{4}\right)$ and equivalent isotropic displacement parameters $\left(\AA^{2} \mathrm{x}\right.$ $10^{3}$ ) for $3+1,4$-butanediol. $U(e q)$ is defined as one third of the trace of the orthogonalized Uij tensor.

\begin{tabular}{lrrrr}
\hline & $\mathrm{X}$ & $\mathrm{y}$ & $\mathrm{Z}$ & $\mathrm{U}(\mathrm{eq})$ \\
\hline $\mathrm{Ir}(1)$ & $5239(1)$ & $3359(1)$ & $8914(1)$ & $10(1)$ \\
$\mathrm{O}(1)$ & $8352(2)$ & $3793(2)$ & $11641(2)$ & $19(1)$ \\
$\mathrm{O}(2)$ & $7271(3)$ & $4690(2)$ & $7219(2)$ & $25(1)$ \\
$\mathrm{O}(3)$ & $3744(2)$ & $2403(2)$ & $9882(2)$ & $16(1)$ \\
$\mathrm{N}(1)$ & $2954(3)$ & $3033(2)$ & $7156(2)$ & $13(1)$ \\
$\mathrm{C}(1)$ & $7163(3)$ & $3608(3)$ & $10601(3)$ & $14(1)$ \\
$\mathrm{C}(2)$ & $6501(3)$ & $4203(3)$ & $7893(3)$ & $16(1)$ \\
$\mathrm{C}(3)$ & $2655(3)$ & $3532(3)$ & $5814(3)$ & $17(1)$ \\
$\mathrm{C}(4)$ & $1081(3)$ & $3322(3)$ & $4730(3)$ & $20(1)$ \\
$\mathrm{C}(5)$ & $-264(3)$ & $2575(3)$ & $5014(3)$ & $19(1)$ \\
$\mathrm{C}(6)$ & $50(3)$ & $2060(3)$ & $6376(3)$ & $16(1)$ \\
$\mathrm{C}(7)$ & $1670(3)$ & $2306(3)$ & $7443(3)$ & $13(1)$ \\
$\mathrm{C}(8)$ & $2095(3)$ & $1805(3)$ & $8977(3)$ & $13(1)$ \\
$\mathrm{C}(9)$ & $799(3)$ & $2413(3)$ & $9741(3)$ & $18(1)$ \\
$\mathrm{C}(10)$ & $555(3)$ & $8791(3)$ & $19(1)$ \\
$\mathrm{O}(4)$ & $2050(4)$ & $2211(2)$ & $2860(2)$ & $23(1)$ \\
$\mathrm{C}(11)$ & $585(3)$ & $3216(3)$ & $18(1)$ \\
$\mathrm{C}(12)$ & $5261(3)$ & $590(3)$ & $4723(3)$ & $17(1)$ \\
\hline & $4389(3)$ & & \\
\hline
\end{tabular}


Table S9. Bond lengths $[\AA]$ and angles $\left[{ }^{\circ}\right]$ for $3+1,4$-butanediol.

\begin{tabular}{ll}
\hline $\mathrm{Ir}(1)-\mathrm{C}(2)$ & $1.839(3)$ \\
$\mathrm{Ir}(1)-\mathrm{C}(1)$ & $1.853(3)$ \\
$\mathrm{Ir}(1)-\mathrm{O}(3)$ & $1.9919(17)$ \\
$\mathrm{Ir}(1)-\mathrm{N}(1)$ & $2.074(2)$ \\
$\mathrm{O}(1)-\mathrm{C}(1)$ & $1.144(3)$ \\
$\mathrm{O}(2)-\mathrm{C}(2)$ & $1.148(3)$ \\
$\mathrm{O}(3)-\mathrm{C}(8)$ & $1.411(3)$ \\
$\mathrm{N}(1)-\mathrm{C}(7)$ & $1.347(3)$ \\
$\mathrm{N}(1)-\mathrm{C}(3)$ & $1.357(3)$ \\
$\mathrm{C}(3)-\mathrm{C}(4)$ & $1.371(4)$ \\
$\mathrm{C}(3)-\mathrm{H}(3)$ & 0.9500 \\
$\mathrm{C}(4)-\mathrm{C}(5)$ & $1.397(4)$ \\
$\mathrm{C}(4)-\mathrm{H}(4)$ & 0.9500 \\
$\mathrm{C}(5)-\mathrm{C}(6)$ & $1.380(3)$ \\
$\mathrm{C}(5)-\mathrm{H}(5)$ & 0.9500 \\
$\mathrm{C}(6)-\mathrm{C}(7)$ & $1.393(3)$ \\
$\mathrm{C}(6)-\mathrm{H}(6)$ & 0.9500 \\
$\mathrm{C}(7)-\mathrm{C}(8)$ & $1.518(3)$ \\
$\mathrm{C}(8)-\mathrm{C}(9)$ & $1.531(3)$ \\
$\mathrm{C}(8)-\mathrm{C}(10)$ & $1.532(3)$ \\
$\mathrm{C}(9)-\mathrm{H}(9 \mathrm{~A})$ & 0.9800 \\
$\mathrm{C}(9)-\mathrm{H}(9 \mathrm{~B})$ & 0.9800 \\
$\mathrm{C}(9)-\mathrm{H}(9 \mathrm{C})$ & 0.9800 \\
$\mathrm{C}(10)-\mathrm{H}(10 \mathrm{~A})$ & 0.9800 \\
$\mathrm{C}(10)-\mathrm{H}(10 \mathrm{~B})$ & 0.9800 \\
$\mathrm{C}(10)-\mathrm{H}(10 \mathrm{C})$ & 0.9800 \\
$\mathrm{O}(4)-\mathrm{C}(11)$ & $1.427(3)$ \\
$\mathrm{O}(4)-\mathrm{H}(4 \mathrm{~A})$ & $0.75(4)$ \\
$\mathrm{C}(11)-\mathrm{C}(12)$ & $1.519(3)$ \\
$\mathrm{C}(11)-\mathrm{H}(11 \mathrm{~A})$ & 0.9900 \\
$\mathrm{C}(11)-\mathrm{H}(11 \mathrm{~B})$ & 0.9900 \\
$\mathrm{C}(12)-\mathrm{C}(12) \# 1$ & $1.526(5)$ \\
$\mathrm{C}(12)-\mathrm{H}(12 \mathrm{~A})$ & 0.9900 \\
$\mathrm{C}(12)-\mathrm{H}(12 \mathrm{~B})$ & 0.9900 \\
& \\
& \\
& \\
& \\
&
\end{tabular}




\begin{tabular}{|c|c|}
\hline$C(2)-\operatorname{Ir}(1)-C(1)$ & $89.00(11)$ \\
\hline $\mathrm{C}(2)-\operatorname{Ir}(1)-\mathrm{O}(3)$ & $175.97(9)$ \\
\hline $\mathrm{C}(1)-\operatorname{Ir}(1)-\mathrm{O}(3)$ & $94.96(9)$ \\
\hline $\mathrm{C}(2)-\operatorname{Ir}(1)-\mathrm{N}(1)$ & $96.33(9)$ \\
\hline $\mathrm{C}(1)-\operatorname{Ir}(1)-\mathrm{N}(1)$ & $174.57(9)$ \\
\hline $\mathrm{O}(3)-\operatorname{Ir}(1)-\mathrm{N}(1)$ & $79.72(8)$ \\
\hline $\mathrm{C}(8)-\mathrm{O}(3)-\operatorname{Ir}(1)$ & $118.22(14)$ \\
\hline $\mathrm{C}(7)-\mathrm{N}(1)-\mathrm{C}(3)$ & $119.3(2)$ \\
\hline $\mathrm{C}(7)-\mathrm{N}(1)-\operatorname{Ir}(1)$ & $114.78(16)$ \\
\hline $\mathrm{C}(3)-\mathrm{N}(1)-\operatorname{Ir}(1)$ & $125.85(17)$ \\
\hline $\mathrm{O}(1)-\mathrm{C}(1)-\operatorname{Ir}(1)$ & $178.6(2)$ \\
\hline $\mathrm{O}(2)-\mathrm{C}(2)-\operatorname{Ir}(1)$ & $177.8(2)$ \\
\hline $\mathrm{N}(1)-\mathrm{C}(3)-\mathrm{C}(4)$ & $122.2(2)$ \\
\hline $\mathrm{N}(1)-\mathrm{C}(3)-\mathrm{H}(3)$ & 118.9 \\
\hline $\mathrm{C}(4)-\mathrm{C}(3)-\mathrm{H}(3)$ & 118.9 \\
\hline$C(3)-C(4)-C(5)$ & $119.1(2)$ \\
\hline $\mathrm{C}(3)-\mathrm{C}(4)-\mathrm{H}(4)$ & 120.4 \\
\hline $\mathrm{C}(5)-\mathrm{C}(4)-\mathrm{H}(4)$ & 120.4 \\
\hline$C(6)-C(5)-C(4)$ & $118.5(2)$ \\
\hline $\mathrm{C}(6)-\mathrm{C}(5)-\mathrm{H}(5)$ & 120.8 \\
\hline $\mathrm{C}(4)-\mathrm{C}(5)-\mathrm{H}(5)$ & 120.8 \\
\hline$C(5)-C(6)-C(7)$ & $120.2(2)$ \\
\hline $\mathrm{C}(5)-\mathrm{C}(6)-\mathrm{H}(6)$ & 119.9 \\
\hline $\mathrm{C}(7)-\mathrm{C}(6)-\mathrm{H}(6)$ & 119.9 \\
\hline $\mathrm{N}(1)-\mathrm{C}(7)-\mathrm{C}(6)$ & $120.7(2)$ \\
\hline $\mathrm{N}(1)-\mathrm{C}(7)-\mathrm{C}(8)$ & $116.3(2)$ \\
\hline$C(6)-C(7)-C(8)$ & $123.1(2)$ \\
\hline $\mathrm{O}(3)-\mathrm{C}(8)-\mathrm{C}(7)$ & $110.04(19)$ \\
\hline $\mathrm{O}(3)-\mathrm{C}(8)-\mathrm{C}(9)$ & $108.1(2)$ \\
\hline $\mathrm{C}(7)-\mathrm{C}(8)-\mathrm{C}(9)$ & $109.8(2)$ \\
\hline $\mathrm{O}(3)-\mathrm{C}(8)-\mathrm{C}(10)$ & $109.5(2)$ \\
\hline$C(7)-C(8)-C(10)$ & $108.8(2)$ \\
\hline $\mathrm{C}(9)-\mathrm{C}(8)-\mathrm{C}(10)$ & $110.6(2)$ \\
\hline $\mathrm{C}(8)-\mathrm{C}(9)-\mathrm{H}(9 \mathrm{~A})$ & 109.5 \\
\hline $\mathrm{C}(8)-\mathrm{C}(9)-\mathrm{H}(9 \mathrm{~B})$ & 109.5 \\
\hline
\end{tabular}




$\begin{array}{ll}\mathrm{H}(9 \mathrm{~A})-\mathrm{C}(9)-\mathrm{H}(9 \mathrm{~B}) & 109.5 \\ \mathrm{C}(8)-\mathrm{C}(9)-\mathrm{H}(9 \mathrm{C}) & 109.5 \\ \mathrm{H}(9 \mathrm{~A})-\mathrm{C}(9)-\mathrm{H}(9 \mathrm{C}) & 109.5 \\ \mathrm{H}(9 \mathrm{~B})-\mathrm{C}(9)-\mathrm{H}(9 \mathrm{C}) & 109.5 \\ \mathrm{C}(8)-\mathrm{C}(10)-\mathrm{H}(10 \mathrm{~A}) & 109.5 \\ \mathrm{C}(8)-\mathrm{C}(10)-\mathrm{H}(10 \mathrm{~B}) & 109.5 \\ \mathrm{H}(10 \mathrm{~A})-\mathrm{C}(10)-\mathrm{H}(10 \mathrm{~B}) & 109.5 \\ \mathrm{C}(8)-\mathrm{C}(10)-\mathrm{H}(10 \mathrm{C}) & 109.5 \\ \mathrm{H}(10 \mathrm{~A})-\mathrm{C}(10)-\mathrm{H}(10 \mathrm{C}) & 109.5 \\ \mathrm{H}(10 \mathrm{~B})-\mathrm{C}(10)-\mathrm{H}(10 \mathrm{C}) & 109.5 \\ \mathrm{C}(11)-\mathrm{O}(4)-\mathrm{H}(4 \mathrm{~A}) & 104(3) \\ \mathrm{O}(4)-\mathrm{C}(11)-\mathrm{C}(12) & 108.6(2) \\ \mathrm{O}(4)-\mathrm{C}(11)-\mathrm{H}(11 \mathrm{~A}) & 110.0 \\ \mathrm{C}(12)-\mathrm{C}(11)-\mathrm{H}(11 \mathrm{~A}) & 110.0 \\ \mathrm{O}(4)-\mathrm{C}(11)-\mathrm{H}(11 \mathrm{~B}) & 110.0 \\ \mathrm{C}(12)-\mathrm{C}(11)-\mathrm{H}(11 \mathrm{~B}) & 110.0 \\ \mathrm{H}(11 \mathrm{~A})-\mathrm{C}(11)-\mathrm{H}(11 \mathrm{~B}) & 108.4 \\ \mathrm{C}(11)-\mathrm{C}(12)-\mathrm{C}(12) \# 1 & 112.2(3) \\ \mathrm{C}(11)-\mathrm{C}(12)-\mathrm{H}(12 \mathrm{~A}) & 109.2 \\ \mathrm{C}(12) \# 1-\mathrm{C}(12)-\mathrm{H}(12 \mathrm{~A}) & 109.2 \\ \mathrm{C}(11)-\mathrm{C}(12)-\mathrm{H}(12 \mathrm{~B}) & 109.2 \\ \mathrm{C}(12) \# 1-\mathrm{C}(12)-\mathrm{H}(12 \mathrm{~B}) & 109.2 \\ \mathrm{H}(12 \mathrm{~A})-\mathrm{C}(12)-\mathrm{H}(12 \mathrm{~B}) & 107.9 \\ & \end{array}$

Symmetry transformations used to generate equivalent atoms:

$\# 1-\mathrm{x}+1,-\mathrm{y},-\mathrm{z}+1$ 
Table S10. Anisotropic displacement parameters $\left(\AA^{2} \times 10^{3}\right)$ for $3+1,4$-butanediol. The anisotropic displacement factor exponent takes the form: $-2 p^{2}\left[h^{2} a^{* 2} U^{11}+\ldots+2 h k a^{*} b^{*}\right.$ $\mathrm{U}^{12}$ ]

\begin{tabular}{lcccccc}
\hline & $\mathrm{U}^{11}$ & $\mathrm{U}^{22}$ & $\mathrm{U} 33$ & $\mathrm{U}^{23}$ & $\mathrm{U} 13$ & $\mathrm{U} 12$ \\
\hline $\mathrm{Ir}(1)$ & $9(1)$ & $11(1)$ & $10(1)$ & $3(1)$ & $3(1)$ & $0(1)$ \\
$\mathrm{O}(1)$ & $14(1)$ & $25(1)$ & $15(1)$ & $2(1)$ & $0(1)$ & $1(1)$ \\
$\mathrm{O}(2)$ & $21(1)$ & $34(1)$ & $23(1)$ & $7(1)$ & $11(1)$ & $-6(1)$ \\
$\mathrm{O}(3)$ & $13(1)$ & $22(1)$ & $12(1)$ & $6(1)$ & $2(1)$ & $-5(1)$ \\
$\mathrm{N}(1)$ & $11(1)$ & $15(1)$ & $12(1)$ & $3(1)$ & $3(1)$ & $-1(1)$ \\
$\mathrm{C}(1)$ & $16(1)$ & $13(1)$ & $16(1)$ & $3(1)$ & $8(1)$ & $2(1)$ \\
$\mathrm{C}(2)$ & $14(1)$ & $19(1)$ & $14(1)$ & $3(1)$ & $2(1)$ & $0(1)$ \\
$\mathrm{C}(3)$ & $15(1)$ & $22(1)$ & $17(1)$ & $8(1)$ & $6(1)$ & $-1(1)$ \\
$\mathrm{C}(4)$ & $20(1)$ & $25(1)$ & $13(1)$ & $8(1)$ & $3(1)$ & $0(1)$ \\
$\mathrm{C}(5)$ & $14(1)$ & $23(1)$ & $16(1)$ & $4(1)$ & $0(1)$ & $-1(1)$ \\
$\mathrm{C}(6)$ & $11(1)$ & $20(1)$ & $17(1)$ & $3(1)$ & $4(1)$ & $-3(1)$ \\
$\mathrm{C}(7)$ & $15(1)$ & $11(1)$ & $13(1)$ & $2(1)$ & $6(1)$ & $-1(1)$ \\
$\mathrm{C}(8)$ & $12(1)$ & $14(1)$ & $12(1)$ & $4(1)$ & $4(1)$ & $-1(1)$ \\
$\mathrm{C}(9)$ & $16(1)$ & $25(1)$ & $16(1)$ & $4(1)$ & $7(1)$ & $1(1)$ \\
$\mathrm{C}(10)$ & $24(1)$ & $14(1)$ & $18(1)$ & $4(1)$ & $6(1)$ & $-1(1)$ \\
$\mathrm{O}(4)$ & $22(1)$ & $28(1)$ & $16(1)$ & $10(1)$ & $2(1)$ & $-3(1)$ \\
$\mathrm{C}(11)$ & $16(1)$ & $25(1)$ & $14(1)$ & $6(1)$ & $3(1)$ & $0(1)$ \\
$\mathrm{C}(12)$ & $15(1)$ & $23(1)$ & $12(1)$ & $3(1)$ & $2(1)$ & $-1(1)$ \\
& & & & & & \\
\hline
\end{tabular}


Table S11. Hydrogen coordinates ( $\left.\times 10^{4}\right)$ and isotropic displacement parameters $\left(\AA^{2} \times 10^{3}\right)$ for 3 + 1,4-butanediol.

\begin{tabular}{lcccl}
\hline & $\mathrm{x}$ & $\mathrm{y}$ & $\mathrm{z}$ & $\mathrm{U}(\mathrm{eq})$ \\
& & & & \\
$\mathrm{H}(3)$ & 3566 & 4045 & 5619 & 21 \\
$\mathrm{H}(4)$ & 908 & 3680 & 3798 & 23 \\
$\mathrm{H}(5)$ & -1370 & 2425 & 4285 & 23 \\
$\mathrm{H}(6)$ & -842 & 1535 & 6587 & 20 \\
$\mathrm{H}(9 \mathrm{~A})$ & 1149 & 2182 & 10770 & 28 \\
$\mathrm{H}(9 \mathrm{~B})$ & -338 & 1924 & 9188 & 28 \\
$\mathrm{H}(9 \mathrm{C})$ & 757 & 3522 & 9753 & 28 \\
$\mathrm{H}(10 \mathrm{~A})$ & 2858 & -315 & 8271 & 28 \\
$\mathrm{H}(10 \mathrm{~B})$ & 889 & -360 & 8206 & 28 \\
$\mathrm{H}(10 \mathrm{C})$ & 2375 & -280 & 9779 & 28 \\
$\mathrm{H}(4 \mathrm{~A})$ & $4810(50)$ & $2230(40)$ & $2050(50)$ & $35(11)$ \\
$\mathrm{H}(11 \mathrm{~A})$ & 4219 & 83 & 2436 & 22 \\
$\mathrm{H}(11 \mathrm{~B})$ & 3249 & 1294 & 3258 & 22 \\
$\mathrm{H}(12 \mathrm{~A})$ & 5750 & 1531 & 5466 & 20 \\
$\mathrm{H}(12 \mathrm{~B})$ & 6552 & 185 & 4639 & 20 \\
& & & & \\
\hline
\end{tabular}


Table S12. Torsion angles $\left[^{\circ}\right]$ for $3+1,4$-butanediol.

\begin{tabular}{lc}
\hline $\mathrm{C}(7)-\mathrm{N}(1)-\mathrm{C}(3)-\mathrm{C}(4)$ & $0.0(4)$ \\
$\mathrm{Ir}(1)-\mathrm{N}(1)-\mathrm{C}(3)-\mathrm{C}(4)$ & $-177.3(2)$ \\
$\mathrm{N}(1)-\mathrm{C}(3)-\mathrm{C}(4)-\mathrm{C}(5)$ & $0.3(4)$ \\
$\mathrm{C}(3)-\mathrm{C}(4)-\mathrm{C}(5)-\mathrm{C}(6)$ & $-0.7(4)$ \\
$\mathrm{C}(4)-\mathrm{C}(5)-\mathrm{C}(6)-\mathrm{C}(7)$ & $1.0(4)$ \\
$\mathrm{C}(3)-\mathrm{N}(1)-\mathrm{C}(7)-\mathrm{C}(6)$ & $0.3(4)$ \\
$\mathrm{Ir}(1)-\mathrm{N}(1)-\mathrm{C}(7)-\mathrm{C}(6)$ & $177.83(18)$ \\
$\mathrm{C}(3)-\mathrm{N}(1)-\mathrm{C}(7)-\mathrm{C}(8)$ & $-179.1(2)$ \\
$\operatorname{Ir}(1)-\mathrm{N}(1)-\mathrm{C}(7)-\mathrm{C}(8)$ & $-1.5(3)$ \\
$\mathrm{C}(5)-\mathrm{C}(6)-\mathrm{C}(7)-\mathrm{N}(1)$ & $-0.7(4)$ \\
$\mathrm{C}(5)-\mathrm{C}(6)-\mathrm{C}(7)-\mathrm{C}(8)$ & $178.5(2)$ \\
$\mathrm{Ir}(1)-\mathrm{O}(3)-\mathrm{C}(8)-\mathrm{C}(7)$ & $-11.1(2)$ \\
$\operatorname{Ir}(1)-\mathrm{O}(3)-\mathrm{C}(8)-\mathrm{C}(9)$ & $-130.99(17)$ \\
$\operatorname{Ir}(1)-\mathrm{O}(3)-\mathrm{C}(8)-\mathrm{C}(10)$ & $108.41(18)$ \\
$\mathrm{N}(1)-\mathrm{C}(7)-\mathrm{C}(8)-\mathrm{O}(3)$ & $7.9(3)$ \\
$\mathrm{C}(6)-\mathrm{C}(7)-\mathrm{C}(8)-\mathrm{O}(3)$ & $-171.4(2)$ \\
$\mathrm{N}(1)-\mathrm{C}(7)-\mathrm{C}(8)-\mathrm{C}(9)$ & $126.8(2)$ \\
$\mathrm{C}(6)-\mathrm{C}(7)-\mathrm{C}(8)-\mathrm{C}(9)$ & $-52.6(3)$ \\
$\mathrm{N}(1)-\mathrm{C}(7)-\mathrm{C}(8)-\mathrm{C}(10)$ & $-112.1(2)$ \\
$\mathrm{C}(6)-\mathrm{C}(7)-\mathrm{C}(8)-\mathrm{C}(10)$ & $68.6(3)$ \\
$\mathrm{O}(4)-\mathrm{C}(11)-\mathrm{C}(12)-\mathrm{C}(12) \# 1$ & $173.6(3)$ \\
\hline & \\
\hline
\end{tabular}

Symmetry transformations used to generate equivalent atoms:

$\# 1-\mathrm{x}+1,-\mathrm{y},-\mathrm{z}+1$ 
Table S13. Hydrogen bonds for $3+1,4$-butanediol [ $\AA$ and $\left.{ }^{\circ}\right]$.

\begin{tabular}{|c|c|c|c|c|}
\hline D-H...A & $\mathrm{d}(\mathrm{D}-\mathrm{H})$ & $\mathrm{d}(\mathrm{H} \ldots \mathrm{A})$ & $\mathrm{d}(\mathrm{D} \ldots \mathrm{A})$ & $<(\mathrm{DHA})$ \\
\hline $\mathrm{C}(4)-\mathrm{H}(4) \ldots \mathrm{O}(2) \# 2$ & 0.95 & 2.55 & $3.279(3)$ & 134.0 \\
\hline $\mathrm{C}(4)-\mathrm{H}(4) \ldots \mathrm{O}(1) \# 3$ & 0.95 & 2.45 & $3.172(3)$ & 132.8 \\
\hline $\mathrm{O}(4)-\mathrm{H}(4 \mathrm{~A}) \ldots \mathrm{O}(3) \# 4$ & $0.75(4)$ & $2.00(4)$ & $2.743(3)$ & $175(4)$ \\
\hline
\end{tabular}

Symmetry transformations used to generate equivalent atoms:

$\# 1-x+1,-y,-z+1 \quad \# 2-x+1,-y+1,-z+1 \quad \# 3 x-1, y, z-1$

$\# 4 \mathrm{x}, \mathrm{y}, \mathrm{z}-1$ 


\section{X-ray crystal structure and data for degradation product $\left[\operatorname{Ir}(\text { pyalc })_{2} \mathbf{I}_{2}\right]$}

Low-temperature diffraction data ( $\omega$-scans) were collected on a Rigaku R-AXIS RAPID diffractometer coupled to an R-AXIS RAPID imaging plate detector with Mo Ka radiation $(\lambda=$ $0.71073 \AA$ ) for the structure of $\operatorname{Ir}(\text { pyalc })_{2} \mathrm{I}_{2}$. The diffraction images were processed and scaled using the Rigaku CrystalClear software (CrystalClear and CrystalStructure; Rigaku/MSC: The $\square$ Woodlands, TX, 2005). The structure was solved with SHELXT and was refined against $F^{2}$ on all data by full-matrix least squares with SHELXL (Sheldrick, G. M. Acta Cryst. 2008, A64, 112-122). All non-hydrogen atoms were refined anisotropically. Hydrogen atoms were included in the model at geometrically calculated positions and refined using a riding model. The isotropic displacement parameters of all hydrogen atoms were fixed to 1.2 times the $U$ value of the atoms to which they are linked (1.5 times for methyl groups). The full numbering scheme of compound $\operatorname{Ir}(\text { pyalc })_{2} \mathrm{I}_{2}$ can be found below. CCDC 1440139 contains the supplementary crystallographic data for this paper. The data can be obtained free of charge from The Cambridge Crystallographic Data Centre. Full details of the X-ray structure determination are in the CIF included as Supporting Information.

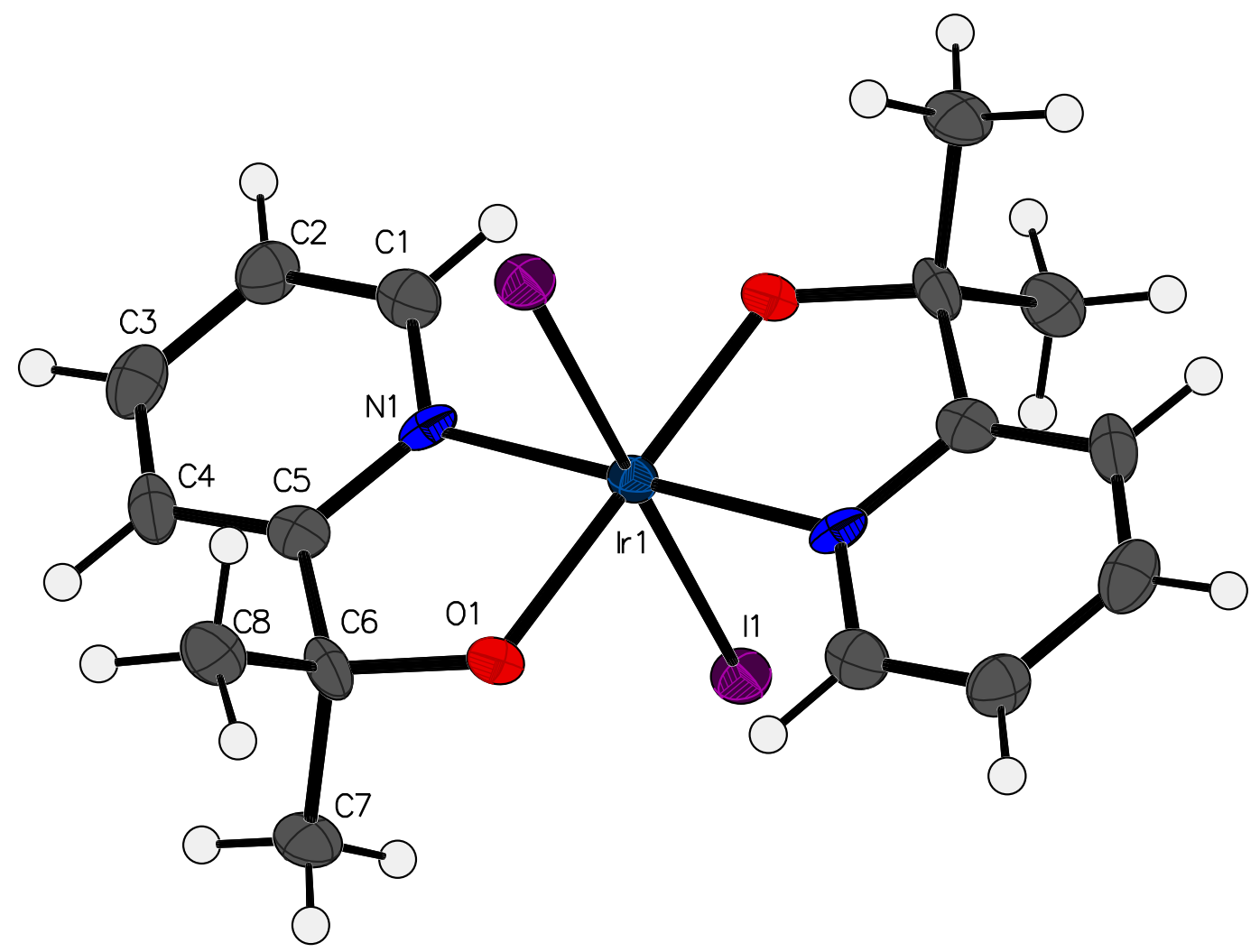

Figure S19. The full numbering scheme of $\operatorname{Ir}(\text { pyalc })_{2} \mathrm{I}_{2}$ with $50 \%$ thermal ellipsoids. The hydrogen atoms are show as arbitrary circles for clarity. Only the asymmetric unit is numbered; the full model is generated by the symmetry operation 1-x, -y, 1-z. 
Table S14. Crystal data and structure refinement for $\operatorname{Ir}(\text { pyalc })_{2} \mathrm{I}_{2}$.

Empirical formula

Formula weight

Temperature

Wavelength

Crystal system

Space group

Unit cell dimensions

Volume

Z

Density (calculated)

Absorption coefficient

$\mathrm{F}(000)$

Crystal size

Crystal color and habit

Diffractometer

$\Theta$ range for data collection

Index ranges

Reflections collected

Independent reflections

Observed reflections $(\mathrm{I}>2 \sigma(\mathrm{I}))$

Completeness to $\theta=25.026^{\circ}$

Absorption correction

Max. and min. transmission

Solution method

Refinement method

Data / restraints / parameters

Goodness-of-fit on $\mathrm{F}^{2}$

Final $R$ indices $[\mathrm{I}>2 \sigma(\mathrm{I})]$

$\mathrm{R}$ indices (all data)

Largest diff. peak and hole
$\mathrm{C}_{16} \mathrm{H}_{20} \mathrm{I}_{2} \mathrm{IrN}_{2} \mathrm{O}_{2}$

718.34

93(2) K

$0.71075 \AA$

Monoclinic

P $21 / \mathrm{n}$

$\mathrm{a}=7.6844(5) \AA \quad \alpha=90^{\circ}$.

$\mathrm{b}=8.7792(6) \AA \quad \beta=100.349(7)^{\circ}$.

$\mathrm{c}=14.3101(10) \AA \quad \gamma=90^{\circ}$.

$949.70(11) \AA^{3}$

2

$2.512 \mathrm{Mg} / \mathrm{m}^{3}$

$10.290 \mathrm{~mm}^{-1}$

658

$0.20 \times 0.10 \times 0.08 \mathrm{~mm}^{3}$

Black Plate

Rigaku R-AXIS RAPID imaging plate

3.280 to $25.026^{\circ}$.

$-9 \leq h \leq 9,-10 \leq k \leq 10,-17 \leq l \leq 16$

8899

$1661[\mathrm{R}(\mathrm{int})=0.0628]$

1496

$99.5 \%$

Semi-empirical from equivalents

1.000 and 0.374

SHELXT-2014/5 (Sheldrick, 2014)

SHELXL-2014/7 (Sheldrick, 2014)

$1661 / 0 / 108$

1.085

$\mathrm{R} 1=0.0321, \mathrm{wR} 2=0.0615$

$\mathrm{R} 1=0.0378, \mathrm{wR} 2=0.0634$

1.486 and -1.049 e. $\AA^{-3}$ 
Table S15. Atomic coordinates ( x 104) and equivalent isotropic displacement parameters $\left(\AA^{2} \mathrm{x}\right.$ $\left.10^{3}\right)$ for $\operatorname{Ir}(\text { pyalc })_{2} I_{2} . U(e q)$ is defined as one third of the trace of the orthogonalized $U^{i j}$ tensor.

\begin{tabular}{llccc}
\hline & \multicolumn{1}{c}{$\mathrm{x}$} & $\mathrm{y}$ & $\mathrm{z}$ & $\mathrm{U}(\mathrm{eq})$ \\
\hline $\mathrm{Ir}(1)$ & 5000 & 0 & 5000 & $16(1)$ \\
$\mathrm{I}(1)$ & $6472(1)$ & $2692(1)$ & $4681(1)$ & $23(1)$ \\
$\mathrm{O}(1)$ & $2867(6)$ & $1091(6)$ & $5165(3)$ & $22(1)$ \\
$\mathrm{N}(1)$ & $5603(7)$ & $343(7)$ & $6407(4)$ & $18(1)$ \\
$\mathrm{C}(5)$ & $4376(9)$ & $1111(8)$ & $6800(5)$ & $21(2)$ \\
$\mathrm{C}(4)$ & $4689(10)$ & $1428(9)$ & $7766(5)$ & $27(2)$ \\
$\mathrm{C}(1)$ & $7103(9)$ & $-124(9)$ & $6978(6)$ & $25(2)$ \\
$\mathrm{C}(2)$ & $7472(10)$ & $185(9)$ & $7932(5)$ & $27(2)$ \\
$\mathrm{C}(3)$ & $6230(10)$ & $990(9)$ & $8329(6)$ & $29(2)$ \\
$\mathrm{C}(6)$ & $2711(9)$ & $1532(9)$ & $6115(5)$ & $23(2)$ \\
$\mathrm{C}(8)$ & $1100(10)$ & $740(10)$ & $6370(6)$ & $31(2)$ \\
$\mathrm{C}(7)$ & $2416(10)$ & $3267(9)$ & $6093(6)$ & $28(2)$ \\
& & & & \\
\hline
\end{tabular}


Table S16. Bond lengths $[\AA]$ and angles $\left[{ }^{\circ}\right]$ for $\operatorname{Ir}(\text { pyalc })_{2} \mathrm{I}_{2}$.

\begin{tabular}{|c|c|}
\hline $\operatorname{Ir}(1)-\mathrm{O}(1) \# 1$ & $1.949(5)$ \\
\hline $\operatorname{Ir}(1)-\mathrm{O}(1)$ & $1.949(5)$ \\
\hline $\operatorname{Ir}(1)-\mathrm{N}(1)$ & $2.006(6)$ \\
\hline $\operatorname{Ir}(1)-N(1) \# 1$ & $2.006(6)$ \\
\hline $\operatorname{Ir}(1)-\mathrm{I}(1) \# 1$ & $2.6938(5)$ \\
\hline $\operatorname{Ir}(1)-\mathrm{I}(1)$ & $2.6938(5)$ \\
\hline $\mathrm{O}(1)-\mathrm{C}(6)$ & $1.438(8)$ \\
\hline $\mathrm{N}(1)-\mathrm{C}(1)$ & $1.351(9)$ \\
\hline $\mathrm{N}(1)-\mathrm{C}(5)$ & $1.361(9)$ \\
\hline$C(5)-C(4)$ & $1.388(10)$ \\
\hline$C(5)-C(6)$ & $1.511(10)$ \\
\hline $\mathrm{C}(4)-\mathrm{C}(3)$ & $1.363(11)$ \\
\hline $\mathrm{C}(4)-\mathrm{H}(4)$ & 0.9500 \\
\hline $\mathrm{C}(1)-\mathrm{C}(2)$ & $1.370(11)$ \\
\hline $\mathrm{C}(1)-\mathrm{H}(1)$ & 0.9500 \\
\hline$C(2)-C(3)$ & $1.388(11)$ \\
\hline $\mathrm{C}(2)-\mathrm{H}(2)$ & 0.9500 \\
\hline $\mathrm{C}(3)-\mathrm{H}(3)$ & 0.9500 \\
\hline$C(6)-C(8)$ & $1.521(10)$ \\
\hline$C(6)-C(7)$ & $1.540(10)$ \\
\hline $\mathrm{C}(8)-\mathrm{H}(8 \mathrm{~A})$ & 0.9800 \\
\hline $\mathrm{C}(8)-\mathrm{H}(8 \mathrm{~B})$ & 0.9800 \\
\hline $\mathrm{C}(8)-\mathrm{H}(8 \mathrm{C})$ & 0.9800 \\
\hline $\mathrm{C}(7)-\mathrm{H}(7 \mathrm{~A})$ & 0.9800 \\
\hline $\mathrm{C}(7)-\mathrm{H}(7 \mathrm{~B})$ & 0.9800 \\
\hline $\mathrm{C}(7)-\mathrm{H}(7 \mathrm{C})$ & 0.9800 \\
\hline $\mathrm{O}(1) \# 1-\operatorname{Ir}(1)-\mathrm{O}(1)$ & 180.0 \\
\hline $\mathrm{O}(1) \# 1-\operatorname{Ir}(1)-\mathrm{N}(1)$ & $98.5(2)$ \\
\hline $\mathrm{O}(1)-\operatorname{Ir}(1)-\mathrm{N}(1)$ & $81.5(2)$ \\
\hline $\mathrm{O}(1) \# 1-\operatorname{Ir}(1)-\mathrm{N}(1) \# 1$ & $81.5(2)$ \\
\hline $\mathrm{O}(1)-\operatorname{Ir}(1)-\mathrm{N}(1) \# 1$ & $98.5(2)$ \\
\hline $\mathrm{N}(1)-\operatorname{Ir}(1)-\mathrm{N}(1) \# 1$ & 180.0 \\
\hline $\mathrm{O}(1) \# 1-\operatorname{Ir}(1)-\mathrm{I}(1) \# 1$ & $88.69(15)$ \\
\hline
\end{tabular}




\begin{tabular}{|c|c|}
\hline $\mathrm{O}(1)-\operatorname{Ir}(1)-\mathrm{I}(1) \# 1$ & $91.31(15)$ \\
\hline $\mathrm{N}(1)-\operatorname{Ir}(1)-\mathrm{I}(1) \# 1$ & $89.42(17)$ \\
\hline $\mathrm{N}(1) \# 1-\operatorname{Ir}(1)-\mathrm{I}(1) \# 1$ & $90.58(17)$ \\
\hline $\mathrm{O}(1) \# 1-\operatorname{Ir}(1)-\mathrm{I}(1)$ & $91.31(15)$ \\
\hline $\mathrm{O}(1)-\operatorname{Ir}(1)-\mathrm{I}(1)$ & $88.69(15)$ \\
\hline $\mathrm{N}(1)-\operatorname{Ir}(1)-\mathrm{I}(1)$ & $90.58(17)$ \\
\hline $\mathrm{N}(1) \# 1-\operatorname{Ir}(1)-\mathrm{I}(1)$ & $89.42(17)$ \\
\hline $\mathrm{I}(1) \# 1-\operatorname{Ir}(1)-\mathrm{I}(1)$ & 180.0 \\
\hline $\mathrm{C}(6)-\mathrm{O}(1)-\operatorname{Ir}(1)$ & $117.5(4)$ \\
\hline $\mathrm{C}(1)-\mathrm{N}(1)-\mathrm{C}(5)$ & $118.6(6)$ \\
\hline $\mathrm{C}(1)-\mathrm{N}(1)-\operatorname{Ir}(1)$ & $125.5(5)$ \\
\hline$C(5)-N(1)-\operatorname{Ir}(1)$ & $115.9(5)$ \\
\hline $\mathrm{N}(1)-\mathrm{C}(5)-\mathrm{C}(4)$ & $120.1(7)$ \\
\hline $\mathrm{N}(1)-\mathrm{C}(5)-\mathrm{C}(6)$ & $114.9(6)$ \\
\hline$C(4)-C(5)-C(6)$ & $124.9(7)$ \\
\hline$C(3)-C(4)-C(5)$ & $120.6(7)$ \\
\hline $\mathrm{C}(3)-\mathrm{C}(4)-\mathrm{H}(4)$ & 119.7 \\
\hline $\mathrm{C}(5)-\mathrm{C}(4)-\mathrm{H}(4)$ & 119.7 \\
\hline $\mathrm{N}(1)-\mathrm{C}(1)-\mathrm{C}(2)$ & $123.0(7)$ \\
\hline $\mathrm{N}(1)-\mathrm{C}(1)-\mathrm{H}(1)$ & 118.5 \\
\hline $\mathrm{C}(2)-\mathrm{C}(1)-\mathrm{H}(1)$ & 118.5 \\
\hline $\mathrm{C}(1)-\mathrm{C}(2)-\mathrm{C}(3)$ & $118.3(7)$ \\
\hline $\mathrm{C}(1)-\mathrm{C}(2)-\mathrm{H}(2)$ & 120.8 \\
\hline $\mathrm{C}(3)-\mathrm{C}(2)-\mathrm{H}(2)$ & 120.8 \\
\hline $\mathrm{C}(4)-\mathrm{C}(3)-\mathrm{C}(2)$ & $119.3(7)$ \\
\hline $\mathrm{C}(4)-\mathrm{C}(3)-\mathrm{H}(3)$ & 120.3 \\
\hline $\mathrm{C}(2)-\mathrm{C}(3)-\mathrm{H}(3)$ & 120.3 \\
\hline $\mathrm{O}(1)-\mathrm{C}(6)-\mathrm{C}(5)$ & $110.1(5)$ \\
\hline $\mathrm{O}(1)-\mathrm{C}(6)-\mathrm{C}(8)$ & $108.3(6)$ \\
\hline$C(5)-C(6)-C(8)$ & $111.3(6)$ \\
\hline $\mathrm{O}(1)-\mathrm{C}(6)-\mathrm{C}(7)$ & $106.5(6)$ \\
\hline$C(5)-C(6)-C(7)$ & $111.0(6)$ \\
\hline$C(8)-C(6)-C(7)$ & $109.5(6)$ \\
\hline $\mathrm{C}(6)-\mathrm{C}(8)-\mathrm{H}(8 \mathrm{~A})$ & 109.5 \\
\hline $\mathrm{C}(6)-\mathrm{C}(8)-\mathrm{H}(8 \mathrm{~B})$ & 109.5 \\
\hline $\mathrm{H}(8 \mathrm{~A})-\mathrm{C}(8)-\mathrm{H}(8 \mathrm{~B})$ & 109.5 \\
\hline
\end{tabular}




$\begin{array}{ll}\mathrm{C}(6)-\mathrm{C}(8)-\mathrm{H}(8 \mathrm{C}) & 109.5 \\ \mathrm{H}(8 \mathrm{~A})-\mathrm{C}(8)-\mathrm{H}(8 \mathrm{C}) & 109.5 \\ \mathrm{H}(8 \mathrm{~B})-\mathrm{C}(8)-\mathrm{H}(8 \mathrm{C}) & 109.5 \\ \mathrm{C}(6)-\mathrm{C}(7)-\mathrm{H}(7 \mathrm{~A}) & 109.5 \\ \mathrm{C}(6)-\mathrm{C}(7)-\mathrm{H}(7 \mathrm{~B}) & 109.5 \\ \mathrm{H}(7 \mathrm{~A})-\mathrm{C}(7)-\mathrm{H}(7 \mathrm{~B}) & 109.5 \\ \mathrm{C}(6)-\mathrm{C}(7)-\mathrm{H}(7 \mathrm{C}) & 109.5 \\ \mathrm{H}(7 \mathrm{~A})-\mathrm{C}(7)-\mathrm{H}(7 \mathrm{C}) & 109.5 \\ \mathrm{H}(7 \mathrm{~B})-\mathrm{C}(7)-\mathrm{H}(7 \mathrm{C}) & 109.5\end{array}$

Symmetry transformations used to generate equivalent atoms:

$\# 1-\mathrm{x}+1,-\mathrm{y},-\mathrm{z}+1$

TableS17. Anisotropic displacement parameters $\left(\AA^{2} \times 10^{3}\right)$ for $\operatorname{Ir}(\text { pyalc })_{2} \mathrm{I}_{2}$. The anisotropic displacement factor exponent takes the form: $-2 p^{2}\left[h^{2} a^{* 2} U^{11}+\ldots+2 h k a^{*} b^{*} U^{12}\right]$

\begin{tabular}{lllllll}
\hline & $\mathrm{U}^{11}$ & $\mathrm{U}^{22}$ & $\mathrm{U}^{33}$ & $\mathrm{U}^{23}$ & $\mathrm{U}^{13}$ & $\mathrm{U}^{12}$ \\
\hline $\mathrm{Ir}(1)$ & $16(1)$ & $15(1)$ & $19(1)$ & $0(1)$ & $4(1)$ & $0(1)$ \\
$\mathrm{I}(1)$ & $24(1)$ & $17(1)$ & $28(1)$ & $1(1)$ & $7(1)$ & $-3(1)$ \\
$\mathrm{O}(1)$ & $22(3)$ & $19(3)$ & $25(3)$ & $2(2)$ & $6(2)$ & $6(2)$ \\
$\mathrm{N}(1)$ & $17(3)$ & $19(3)$ & $17(3)$ & $8(3)$ & $-2(2)$ & $0(3)$ \\
$\mathrm{C}(5)$ & $20(4)$ & $14(4)$ & $30(5)$ & $0(3)$ & $6(3)$ & $-5(3)$ \\
$\mathrm{C}(4)$ & $33(4)$ & $26(5)$ & $24(4)$ & $-5(3)$ & $15(4)$ & $-4(4)$ \\
$\mathrm{C}(1)$ & $22(4)$ & $23(5)$ & $32(5)$ & $0(3)$ & $7(3)$ & $-1(3)$ \\
$\mathrm{C}(2)$ & $31(4)$ & $26(5)$ & $21(4)$ & $-1(3)$ & $-1(3)$ & $-1(3)$ \\
$\mathrm{C}(3)$ & $37(5)$ & $28(5)$ & $23(4)$ & $0(3)$ & $6(4)$ & $-11(4)$ \\
$\mathrm{C}(6)$ & $22(4)$ & $23(4)$ & $27(4)$ & $-5(3)$ & $14(3)$ & $0(3)$ \\
$\mathrm{C}(8)$ & $25(4)$ & $33(5)$ & $35(5)$ & $1(4)$ & $9(4)$ & $5(4)$ \\
$\mathrm{C}(7)$ & $36(5)$ & $15(4)$ & $31(5)$ & $-4(3)$ & $5(4)$ & $1(3)$ \\
& & & & & & \\
\hline
\end{tabular}


Table S18. Hydrogen coordinates ( $\left.\mathrm{x} 10^{4}\right)$ and isotropic displacement parameters $\left(\AA^{2} \mathrm{x} 10^{3}\right)$ for $\operatorname{Ir}(\text { pyalc })_{2} \mathrm{I}_{2}$.

$\begin{array}{llll}\mathrm{x} & \mathrm{y} & \mathrm{U} & \mathrm{eq})\end{array}$

$\begin{array}{lrrll}\mathrm{H}(4) & 3821 & 1955 & 8037 & 32 \\ \mathrm{H}(1) & 7939 & -692 & 6706 & 30 \\ \mathrm{H}(2) & 8550 & -144 & 8312 & 32 \\ \mathrm{H}(3) & 6453 & 1233 & 8987 & 35 \\ \mathrm{H}(8 \mathrm{~A}) & 49 & 1008 & 5903 & 46 \\ \mathrm{H}(8 \mathrm{~B}) & 936 & 1067 & 7003 & 46 \\ \mathrm{H}(8 \mathrm{C}) & 1276 & -365 & 6368 & 46 \\ \mathrm{H}(7 \mathrm{~A}) & 3327 & 3762 & 5800 & 41 \\ \mathrm{H}(7 \mathrm{~B}) & 2488 & 3646 & 6743 & 41 \\ \mathrm{H}(7 \mathrm{C}) & 1245 & 3499 & 5722 & 41 \\ \end{array}$


Table S19. Torsion angles $\left[^{\circ}\right]$ for $\operatorname{Ir}(\text { pyalc })_{2} \mathrm{I}_{2}$.

\begin{tabular}{lc}
\hline $\mathrm{C}(1)-\mathrm{N}(1)-\mathrm{C}(5)-\mathrm{C}(4)$ & $1.2(10)$ \\
$\mathrm{Ir}(1)-\mathrm{N}(1)-\mathrm{C}(5)-\mathrm{C}(4)$ & $-178.8(5)$ \\
$\mathrm{C}(1)-\mathrm{N}(1)-\mathrm{C}(5)-\mathrm{C}(6)$ & $-177.6(6)$ \\
$\mathrm{Ir}(1)-\mathrm{N}(1)-\mathrm{C}(5)-\mathrm{C}(6)$ & $2.3(8)$ \\
$\mathrm{N}(1)-\mathrm{C}(5)-\mathrm{C}(4)-\mathrm{C}(3)$ & $0.7(11)$ \\
$\mathrm{C}(6)-\mathrm{C}(5)-\mathrm{C}(4)-\mathrm{C}(3)$ & $179.4(7)$ \\
$\mathrm{C}(5)-\mathrm{N}(1)-\mathrm{C}(1)-\mathrm{C}(2)$ & $-2.2(11)$ \\
$\operatorname{Ir}(1)-\mathrm{N}(1)-\mathrm{C}(1)-\mathrm{C}(2)$ & $177.8(6)$ \\
$\mathrm{N}(1)-\mathrm{C}(1)-\mathrm{C}(2)-\mathrm{C}(3)$ & $1.2(12)$ \\
$\mathrm{C}(5)-\mathrm{C}(4)-\mathrm{C}(3)-\mathrm{C}(2)$ & $-1.7(12)$ \\
$\mathrm{C}(1)-\mathrm{C}(2)-\mathrm{C}(3)-\mathrm{C}(4)$ & $0.8(11)$ \\
$\mathrm{Ir}(1)-\mathrm{O}(1)-\mathrm{C}(6)-\mathrm{C}(5)$ & $3.6(7)$ \\
$\operatorname{Ir}(1)-\mathrm{O}(1)-\mathrm{C}(6)-\mathrm{C}(8)$ & $-118.2(5)$ \\
$\operatorname{Ir}(1)-\mathrm{O}(1)-\mathrm{C}(6)-\mathrm{C}(7)$ & $124.1(5)$ \\
$\mathrm{N}(1)-\mathrm{C}(5)-\mathrm{C}(6)-\mathrm{O}(1)$ & $-3.8(9)$ \\
$\mathrm{C}(4)-\mathrm{C}(5)-\mathrm{C}(6)-\mathrm{O}(1)$ & $177.4(7)$ \\
$\mathrm{N}(1)-\mathrm{C}(5)-\mathrm{C}(6)-\mathrm{C}(8)$ & $116.3(7)$ \\
$\mathrm{C}(4)-\mathrm{C}(5)-\mathrm{C}(6)-\mathrm{C}(8)$ & $-62.5(10)$ \\
$\mathrm{N}(1)-\mathrm{C}(5)-\mathrm{C}(6)-\mathrm{C}(7)$ & $-121.4(7)$ \\
$\mathrm{C}(4)-\mathrm{C}(5)-\mathrm{C}(6)-\mathrm{C}(7)$ & $59.8(9)$ \\
\hline & \\
\hline
\end{tabular}

Symmetry transformations used to generate equivalent atoms:

\#1-x+1,-y,-z+1 